\title{
Removal of GABA within Adult Modulatory Systems Alters Electrical Coupling and Allows Expression of an Embryonic-Like Network
}

\author{
Eric Ducret, ${ }^{\star}$ Yves Le Feuvre, ${ }^{\star}$ Pierre Meyrand, and Valérie S. Fénelon \\ Laboratoire de Neurobiologie des Réseaux, Université Bordeaux I and Centre National de la Recherche Scientifique, Unité Mixte de Recherche 5816, 33405 \\ Talence, France
}

\begin{abstract}
The maturation and operation of neural networks are known to depend on modulatory neurons. However, whether similar mechanisms may control both adult and developmental plasticity remains poorly investigated. To examine this issue, we have used the lobster stomatogastric nervous system (STNS) to investigate the ontogeny and role of GABAergic modulatory neurons projecting to small pattern generating networks. Using immunocytochemistry, we found that modulatory input neurons to the stomatogastric ganglion (STG) express GABA only after metamorphosis, a time that coincides with the developmental switch from a single to multiple pattern generating networks within the STNS. We demonstrate that blocking GABA synthesis with 3-mercapto-propionic acid within the adult modulatory neurons results in the reconfiguration of the distinct STG networks into a single network that generates a unified embryoniclike motor pattern. Using dye-coupling experiments, we also found that gap-junctional coupling is greater in embryos and GABAdeprived adults exhibiting the unified motor pattern compared with control adults. Furthermore, GABA was found to diminish directly the extent and strength of electrical coupling within adult STG networks. Together, these observations suggest the acquisition of a GABAergic phenotype by modulatory neurons after metamorphosis may induce the reconfiguration of the single embryonic network into multiple adult networks by directly decreasing electrical coupling. The findings also suggest that adult neural networks retain the ability to express typical embryonic characteristics, indicating that network ontogeny can be reversed and that changes in electrical coupling during development may allow the segregation of multiple distinct functional networks from a single large embryonic network.
\end{abstract}

Key words: stomatogastric nervous system; network reconfiguration; development; 3-mercapto-propionic acid; neurobiotin; motor pattern

\section{Introduction}

The role of neuromodulatory inputs in controlling the operation and maturation of neural networks has been studied extensively. In the adult, neuromodulatory inputs are known to exert shortterm effects on target cells by transiently altering cellular and synaptic properties, thereby allowing adaptive plasticity (HarrisWarrick and Marder, 1991). In extreme cases, modulatory mechanisms can induce network reconfigurations that lead to the complete reorganization of network outputs (Meyrand et al., 1991). During ontogeny, neuromodulatory inputs are thought to be responsible for the progressive acquisition (Pflüger, 1999; Marder and Rehm, 2005) and long-term maintenance (Mizrahi et al., 2001) of cellular and synaptic properties by networks. However, whether rapid modulatory phenomena such as network reconfiguration may play a role in the switch from immature to mature network operation remains poorly investigated.

\footnotetext{
Received June 21, 2006; revised Jan. 16, 2007; accepted Jan. 16, 2007.

This work was supported by Action Concertée Incitative "Jeune Chercheur" to V.S.F. and a doctoral student fellowship to E.D., both from the Ministère de la Recherche et de la Technologie. We deeply thank Dr. A. E. Herbison for critical comments and careful editing of a previous version of this manuscript.

*E.D. and Y.L.F. contributed equally to this work.

Correspondence should be addressed to Dr. Yves Le Feuvre at the above address. E-mail: Yves.Lefeuvre@bordeaux.inserm.fr.

DOI:10.1523/JNEUROSCI.4406-06.2007

Copyright $\odot 2007$ Society for Neuroscience $\quad$ 0270-6474/07/273626-13\$15.00/0
}

The crustacean stomatogastric nervous system (STNS) is a particularly useful model system for examining the role of neuromodulatory inputs in regulating adult and developmental network plasticity. Thirty neurons located in the stomatogastric ganglion (STG) (Fenelon et al., 1998) are organized into a single motor network in embryos and premetamorphic larvae but form three distinct motor networks in postmetamorphic animals (Casasnovas and Meyrand, 1995). The expression of these different networks is controlled by the same group of modulatory neurons located in more rostral ganglia in embryos and adults (Le Feuvre et al., 1999, 2001). The GABAergic component of this modulatory input is well characterized and is known to consist of four identified pairs of neurons (Cournil et al., 1990). Previous studies have shown that some of these modulatory GABAergic neurons, in addition to exogenous GABA, can exert important effects on the adult STNS networks (Cazalets et al., 1987; Nagy et al., 1994; Faumont, 1999; Meyrand et al., 2000; Swensen et al., 2000). A recent developmental study has shown that these modulatory neurons are present in the embryo but at least four of them do not express GABA at this developmental stage (Le Feuvre et al., 2001).

In the present study, we examined the ontogeny of modulatory GABAergic inputs and assessed their role in the development of STG networks. We found that GABA is expressed by neuro- 
modulatory inputs after metamorphosis at the time when the adult STG network patterns become evident. Pharmacological blockade of GABA synthesis within adult neuromodulatory inputs results in the reconfiguration of the three adult STG networks into a single network producing a unified embryonic-like motor pattern. Dye-coupling within the STG was found to be weaker in control adults expressing three motor patterns compared with embryos and GABA-deprived adults that express a single motor pattern, and GABA appears to directly reduce gapjunctional coupling within the STG. Together, these findings suggest that developmental plasticity may result from the reversible, short-term reconfiguration of target networks, and that changes in electrical coupling may play a key role in specifying multiple distinct networks from a single embryonic network.

\section{Materials and Methods}

Animals and dissections. Experiments were performed on embryonic $(n=50)$, larval $(n=21)$, juvenile $(n=13)$, and adult $(n=60)$ European lobsters (Homarus gammarus). Male adults and egg-bearing females were obtained from the local fishery supply, whereas larvae and juveniles were purchased from South Wexford Lobster Co-operative Society (Dublin, Ireland). Animals were kept in individual aquariums filled with $15^{\circ} \mathrm{C}$ aerated circulating artificial seawater. Determination of embryonic and larval stages was performed as described previously (Fenelon et al., 1998). Briefly, the percent staging system based on eye index (Helluy and Beltz, 1991) was used to determine the developmental stage of the embryos, ranging in this study from 60 to $100 \%$ development. Morphological features of the abdomen and appendages were used to distinguish between larval stages I, II, III, and IV. Juvenile animals used for experiments had cephalothorax lengths (from the anterior point of the rostrum to the posterior edge of the thorax) ranging from 8.5 to $10 \mathrm{~mm}$.

Dissections were performed in aerated Panulirus saline [containing the following (in mM): $479.12 \mathrm{NaCl}, 12.74 \mathrm{KCl}, 13.2 \mathrm{CaCl}_{2}, 10 \mathrm{MgSO}_{4}$, $3.2 \mathrm{Na}_{2} \mathrm{SO}_{4}$, and 5 HEPES, pH 7.45]. Adult, larval, and embryonic STNS were dissected as described previously (Casasnovas and Meyrand, 1995; Le Feuvre et al., 2001).

Electrophysiology. Stomatogastric nervous systems were pinned onto sylgard-coated Petri dishes and constantly perfused with chilled Panulirus saline. Intracellular recordings were performed using conventional sharp electrodes filled with $2 \mathrm{M} \mathrm{K}$-acetate (15-40 M $\Omega$ ), either in the soma (adults) or in the target muscles (embryos) of STG neurons. All membrane potentials were measured at the most hyperpolarized point (bottom) of the cell oscillation. On 42 neurons, belonging to seven different types of neurons, membrane potentials ranged from -35 to $-95 \mathrm{mV}$ $(70.5 \pm 3.1 \mathrm{mV})$. More than two-thirds of neurons had membrane potentials in the range of -50 and $-80 \mathrm{mV}$. For each experiment and each neuron, the voltage variation between the onset and the end of the experiment was calculated. The mean value of these variations was $1.75 \pm$ $1.32 \mathrm{mV}$. The potentials were therefore usually quite stable throughout a given experiment. Intracellular signals were amplified using an Axoclamp 2B (Molecular Devices, Union City, CA) and digitized using a CED device (Cambridge Electronic Design, Cambridge, UK). Data acquisition and analysis were performed using Spike2 software. For phase relationship computation, the event times were extracted using Spike2 software peak detection procedure. In the case of extracellular channels where more than one different pool of neurons could be recorded, the size and the shape of the spike were used to identify the neurons.

Electrical coupling characterization. To measure coupling coefficients, pairs of pyloric dilator (PD) neurons were isolated from modulatory inputs and antagonist network partners by superperfusing a mixture of tetrodotoxin (TTX; $10^{-7} \mathrm{M}$ ) and picrotoxin (PTX; $10^{-6} \mathrm{M}$ ). Two electrodes were impaled in each of the two PD neurons: one for current injection, the other for voltage measurement. Brief $(500 \mathrm{~ms})$ current pulses (range, -20 to $+20 \mathrm{nA}, 4 \mathrm{nA}$ steps, 10 times each) were injected into one PD neuron and the voltage deflection measured both in the presynaptic (injected) and postsynaptic PD neurons. The mean of 10 voltage deflections for each value of injected current was used to con- struct input/output curves. Coupling coefficient is defined as the slope of the curve (in its linear part) representing postsynaptic voltage variation as a function of presynaptic voltage variation. The input resistance is calculated as the slope of the $V-I$ curve. To compare between experiments, all data were normalized using the mean of control input resistance or control coupling coefficient. Measurements were made after $30-45 \mathrm{~min}$ application of drugs and $1-2 \mathrm{~h}$ after washing out GABA for recovery.

Dye-coupling experiments. A low molecular weight tracer, Neurobiotin ( $\mathrm{N}$-(2-aminoethyl) biotinamide hydrochloride $(323 \mathrm{~g} / \mathrm{mol}$, diluted $10 \%$ in $2 \mathrm{M}$ potassium acetate; Vector Laboratories, Burlingame, CA) was injected into identified $\mathrm{PD}$ neurons using positive current injection (1 $\mathrm{Hz}, 1-3 \mathrm{nA}, 500 \mathrm{~ms}, 30-60 \mathrm{~min})$. PD neurons were identified using conventional electrophysiological techniques: each spike in the soma is followed with a constant latency spike recorded in the PD nerve (adult) or by a constant latency excitatory junctional potential recorded in PD muscle (embryo). Neurobiotin is known to diffuse through the pores of electrical synapses and therefore diffuse to electrically coupled neurons. This procedure enables the visualization of the population of neurons dye coupled to an individual PD neuron. Neurobiotin was allowed to diffuse overnight before fixation of preparations in $4 \%$ paraformaldehyde (PFA) in $0.1 \mathrm{~m} \mathrm{PBS}, \mathrm{pH} 7.4,115 \mathrm{~mm} \mathrm{NaCl}$, and $4 \mathrm{~mm} \mathrm{KCl}$ for 10-12 $\mathrm{h}$ at $4^{\circ} \mathrm{C}$. They were then rinsed five times over at least $2 \mathrm{~h}$ with PBS, incubated at $4^{\circ} \mathrm{C}$ for $24 \mathrm{~h}$ in Avidine DCS-fluoresceine [1:200 in PBS with $0.3 \%$ Triton X-100 (PBST); Vector Laboratories], and finally rinsed five times in PBS before in toto acquisition on a confocal microscope.

Immunocytochemistry. Preparations were fixed in 4\% PFA for $2 \mathrm{~h}$ at $4^{\circ} \mathrm{C}$ and then rinsed several times in PBST. Primary antibody (polyclonal anti-GABA raised in rabbit; Sigma, St. Louis, MO) was diluted at 1:200 in PBST with $10 \%$ normal goat serum (NGS) and incubated with preparations for $48 \mathrm{~h}$ at $4^{\circ} \mathrm{C}$. After numerous washes in PBST, preparations were incubated in anti-rabbit FITC-conjugated Igs raised in goat (1:200 in PBST, 10\% NGS; Sigma) for $12-24 \mathrm{~h}$ at $4^{\circ} \mathrm{C}$ and then rinsed in PBS before in toto acquisition on a confocal microscope. Preadsorption controls with this anti-GABA antibody had been performed previously in the STNS of a related species, Cancer borealis (Blitz et al.,1999). However, to verify the binding specificity in the lobster, GABA antibody was preincubated with $10^{-4} \mathrm{M}$ GABA at $4^{\circ} \mathrm{C}$ overnight before immunohistochemical staining. In these conditions, no staining was observed $(n=1)$. In addition, using the same antibody and protocol, it has been shown by HPLC analysis that GABA immunoreactivity represents the presence of GABA in the related species C. borealis (Swensen et al., 2000).

Confocal microscopy. Preparations were viewed either directly in the Petri dish or after mounting in $90 \%$ glycerol- $10 \% \mathrm{NaHCO}_{3}$ with a Leica (Nussloch, Germany) TCS 4D or a BX51 Olympus (Tokyo, Japan) Fluoview 500 laser scanning confocal microscope. Twenty to 60 optical sections of 1-3 $\mu \mathrm{m}$ thickness were typically acquired for each preparation.

Quantification of dye-coupled neurons. To quantify the number of PD dye-coupled cells, individual stacks were loaded into the Scion (Frederick, MD) Image software and converted to gray scale. For each neuronal soma, the mean gray level was measured in one optical slice taken at the largest diameter of the neuron. Background was measured in three zones of equal areas in the middle of STG in a given stack and the mean was subtracted from the values measured for the cell bodies. This allowed us to unambiguously count the number of stained somata.

Pharmacology. Unless specified, all chemicals were purchased from Sigma and diluted in standard Panulirus saline. GABA, TTX, and PTX were bath applied to the entire STNS preparation. To block GABA synthesis within our preparation, $1 \mathrm{~mm}$ 3-mercapto-propionic acid (3MPA), a competitive inhibitor of glutamate decarboxylase (the GABA synthesis enzyme) was used (Lamar, 1970). This substance was shown to reduce GABA levels (Karlsson et al., 1974; Löscher et al., 1985; Herbison et al., 1990) and GABAergic synaptic transmission (Golan and Grossman, 1996; Pearlstein et al., 1998) in various preparations without affecting other neurotransmitters such as glutamate and aspartate (Kapetanovic et al., 1988). A Vaseline well surrounding the ganglia of interest (anterior ganglia or STG) was built and used to superfuse only part of the preparation with Ringer containing 3-MPA. 


\section{Results}

Acquisition of GABA in the developing lobster nervous system

The development of the lobster occurs in three main phases: embryonic, larval, and postmetamorphic. The embryonic phase, which occurs in the egg is followed by three larval stages [first (LI), second (LII), and third (LIII) larval stages]; the metamorphic moult that occurs at the end of third larval stage leads to postmetamorphic animals [fourth larval stage (LIV), juveniles, and adults]. There was no previous evidence that GABA was expressed within the embryonic and larval CNS of the European lobster. We therefore addressed whether GABA was expressed in the developing CNS. Immunocytochemical detection of GABA in the brain and ventral nerve cord revealed that this neurotransmitter was widely expressed in the CNS of the European lobster throughout embryonic $(n=9)$, larval $(n=16)$, and juvenile stages $(n=3)$ (Fig. 1$)$. Indeed, numerous intensely stained somata could be observed in the cerebroïd, thoracic, and abdominal ganglia at all developmental stages.

In this study, we concentrated our immunocytochemical detection of GABA to the developing STNS. The STNS consists of four interconnected ganglia: the STG, the esophageal ganglion (OG) and the paired commissural ganglia (CoGs) (Fig. $2 A 1$, schematic drawings of embryonic and larval STNS, A2, juvenile and adult STNS). The somata of most modulatory neurons projecting to the STG are located within the OG and CoGs (Le Feuvre et al., 2001). Using immunocytochemistry, we found that GABA was expressed in the CoGs from early in development [as early as embryonic day 60 (E60)] through to adulthood (Fig. $2 B$, top). Within the embryonic $(n=6)$, larval (LI-LIV; $n=21)$, juvenile $(n=13)$, and adult $(n=13)$ stages, numerous somata and fibers within the CoG were immunopositive for GABA (see labels on juvenile CoGs micrograph). In contrast, no GABA staining was observed in the embryonic $(n=9)$ and LI to LIII $(n=12)$ STG. As reported previously in the adult (Cazalets et al.,1987; Cournil et al., 1990), a faint neuropilar GABA staining was visible in the anterior part of the STG of juveniles and adults ( $n=5$ of 10 and 5 of 6 , respectively) (Fig. $2 B$, middle, $D$, top). Strong GABA immunoreactivity within axons entering the STG was only observed in one adult preparation (Fig. $2 B$, box in adult STG micrograph). In the fourth larval stage that follows metamorphosis, GABAergic neuropilar staining was observed in the anterior part of two of eight STGs (Fig. $2 B$, middle, $D$ ).

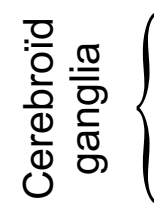

\section{Embryo LIV Juvenile}
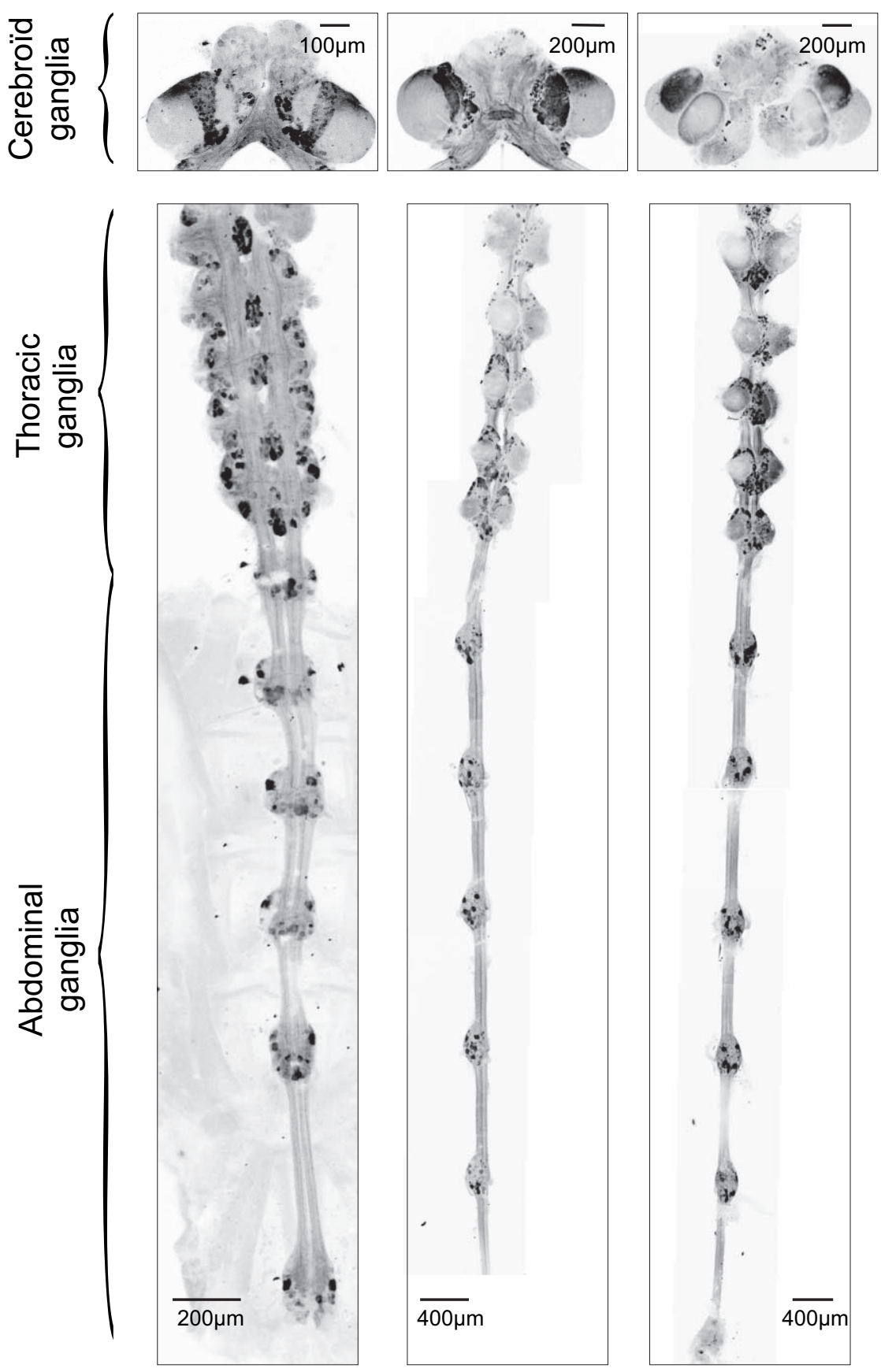

Figure 1. GABA is expressed early in development throughout the brain and the ventral nerve cord of $H$. gammarus. Immunocytochemical detection of GABA within the cerebroïd ganglia (top) and the lower part of ventral nerve cord (the 5 last thoracic ganglia and the 6 abdominal ganglia; bottom) of embryonic (E85; left), larval (middle; fourth larval stage) and juvenile (right) animals reveal the presence of numerous GABA-immunoreactive clusters of cells and neuropil that can be observed as early as midembryonic development. Reconstructed images were obtained by aligning maximal projections of confocal stacks containing $20-40$ optical sections. For embryo and LIV, brain and nerve cord are from different preparations. Note that the sixth abdominal ganglion is missing in the LIV.

Because all GABAergic neurons within the OG project to the STG in adults (Cournil et al., 1990), we investigated the chronology of GABA expression within the OG throughout development. No staining was observed in $75 \%$ of premetamorphic animals (embryos and LI-LIII) (Fig. $2 B$, bottom). In the remaining 


\section{A1}
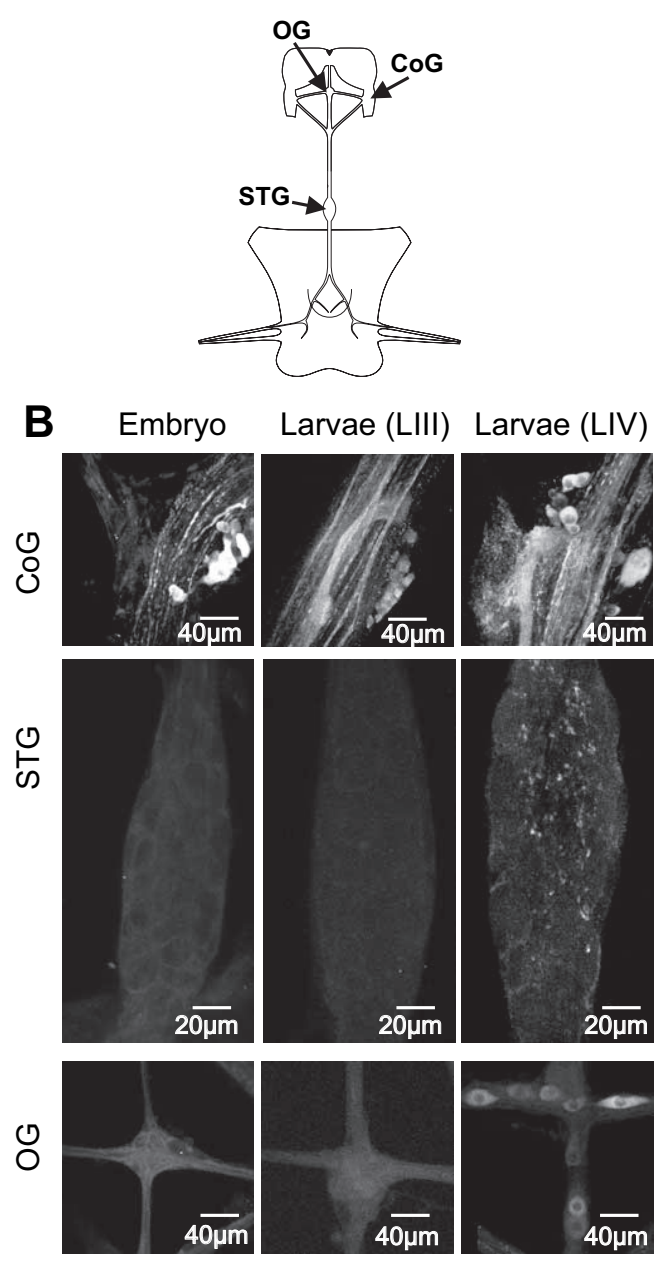

C

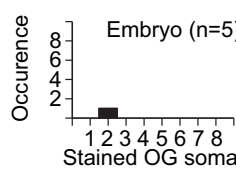

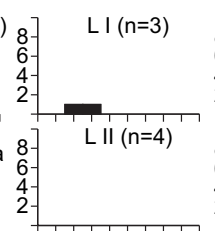

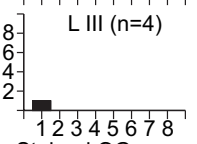

Stained OG som
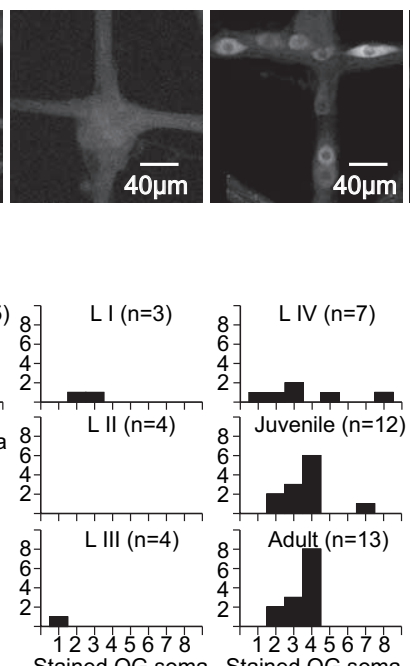

A2
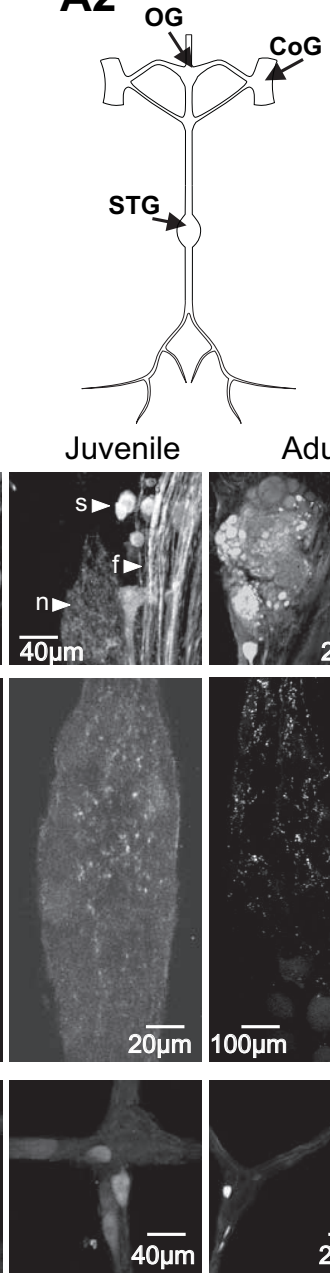

D

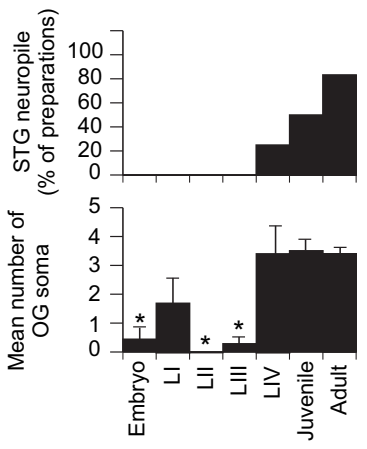

Figure 2. GABA appears within the modulatory input system to the STG after metamorphosis. $\boldsymbol{A} 1, \boldsymbol{A 2}$, Position of the main STNS ganglia depicted on schematic drawings of the embryonic (A1) and adult (A2) STNS. B, Microphotographs of GABA immunoreactivity in the COG (top), STG (middle), and OG (bottom) observed at different developmental stages. Note the intense staining in the CoG of embryonic, larval, and postmetamorphic (LIV, juvenile, and adult) stages. In juvenile CoG, letters indicate the presence of stained neuropil ( $\mathrm{n}$ ), somata (s), and fibers ( $\mathrm{f}$ ). GABA staining can only be detected in the STG after metamorphosis. Indeed no staining is visible in the STG in premetamorphic (embryo, LIII) animals whereas in postmetamorphic animals, a faint neuropil is visible within the most anterior part of the STG. Note that the inset in the adult STG micrograph illustrates strongly stained fibers entering an other STG (see Results, Acquisition of GABA in the developing lobster nervous system). Scale bar, $100 \mu \mathrm{m}$. Similarly, embryonic and larval (LIII) OG usually display no GABA-immunoreactive cells whereas, typically, several soma are stained in the LIV, juvenile, and adult OG. All images are maximal projections of 20-40 optical sections. $\boldsymbol{C}$, Distribution of the number of OG-stained soma for each developmental stage. $\boldsymbol{D}$, Diagrams summarizing the chronology of appearance of GABA within the modulatory input system to the STG. The top panel shows the percentage of preparations in which a stained neuropil was observed within the STG for each developmental stage. The bottom panel shows the mean number of stained soma in the OG for each developmental stage (mean \pm SEM). Note that the mean number of GABAergic OG soma in embryo, LII, and LIII is statistically different from the mean number observed in adult ( ${ }^{*} p<0.005$, one way ANOVA followed by Mann-Whitney test), whereas no statistically significant difference can be observed for $\operatorname{LIOG}(p=0.05)$.

preparations (4 of 16), a few (one to three) very faintly labeled cells were observed (Fig. 2C). In contrast, one to eight labeled cells were consistently observed in the LIV OG ( $n=6$ of 7 ) (Fig. $2 B$, bottom, $C$ ). All juvenile $(n=12)$ and adult $(n=13)$ OG typically displayed up to four stained soma (Fig. $2 B, C$ ). Statistical analysis revealed no significant difference between adult, juvenile, and LIV number of OG-stained soma. In contrast, although the number of stained OG soma in LI was not statistically different from the adult (Mann-Whitney test, $p=0.05$ ), the number of embryonic, LII, and LIII OG-stained soma was significantly lower than in the adult $(p<0.005$, one way ANOVA followed by MannWhitney test). Together, our results show that GABA is definitively acquired within the modulatory inputs to the STG after metamorphosis (summarized in Fig. 2D, top and bottom for STG and OG, respectively).

\section{Effects of bath-applied GABA on the embryonic STNS}

The appearance of GABA within modulatory inputs to the STG coincides with the emergence of the three adult networks from the single premetamorphic network (Casasnovas and Meyrand, 1995). We therefore addressed whether bath-applied GABA was able to induce the expression of the adult network phenotypes within the embryonic STNS.

We used bath application of GABA (concentration range, $10^{-5}$ to $10^{-3} \mathrm{M}$ ) to address the effect of GABA on the rhythmic motor patterns expressed by embryonic preparations. Bath application of GABA to the whole embryonic STNS resulted within $2 \mathrm{~min}$ in the complete cessation of the single embryonic rhythm $(n=$ 7 of 8 preparations; in the remaining preparation rhythmic activity was still observed in one pyloric muscle). This effect was reversible within 10-20 min of rinsing (Fig. 3A). GABA receptors are therefore present and functional within the embryonic STNS before the detection of GABA within neuromodulatory input fibers. To determine whether the inhibition of the embryonic network results from a direct action of GABA on STG neurons, or from an indirect action through an inhibition of modulatory input neurons that control STG neurons, we performed selective application of GABA to the STG alone or to the more rostral ganglia (OG, CoGs, and cerebroïd ganglia) (Fig. 2A1, drawing). GABA applied directly to the STG induced a reversible loss of rhythmic activities $(n=$ 4 of 4 ) (Fig. 3B). Similarly, when GABA 
A

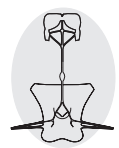

B

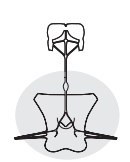

C

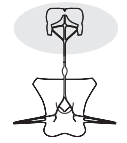

Control

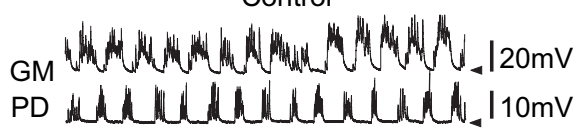

$10^{-5} \mathrm{M}$ GABA on whole preparation
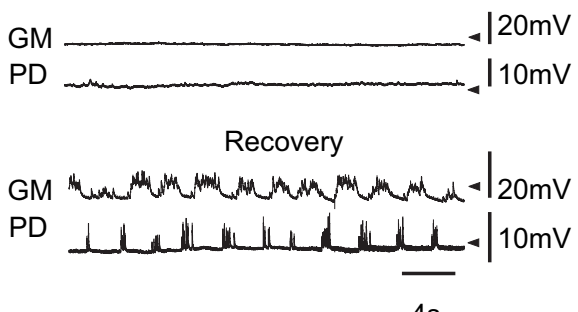

$4 \mathrm{~s}$

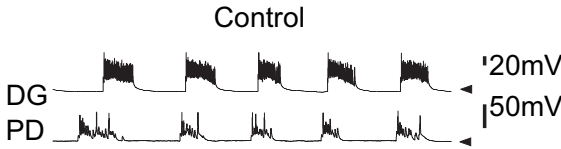

$10^{-3} \mathrm{M}$ GABA on STG
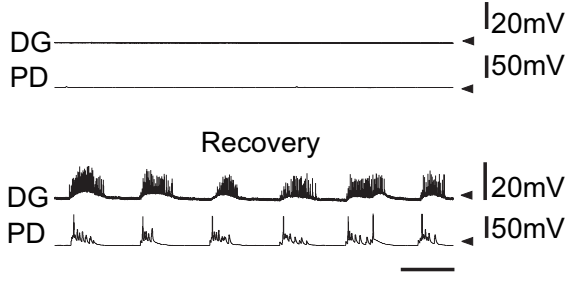

$4 \mathrm{~s}$

Control

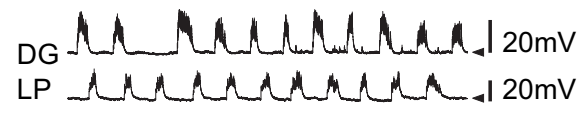

$5.10^{-5} \mathrm{M}$ GABA on CoGs and OG

$D G \longrightarrow-120 \mathrm{mV}$ LP $20 \mathrm{mV}$

Figure 3. Bath-applied GABA inhibits the expression of spontaneous embryonic motor pattern. $\boldsymbol{A}-\boldsymbol{C}$, Superfusion of GABA on the whole STNS preparation $(\boldsymbol{A})$, on the STG alone $(\boldsymbol{B})$, or on the anterior ganglia alone $(C)$ results in a complete cessation of the spontaneous embryonic rhythm monitored here through intramuscular recordings of pyloric muscles (PD, LP) and gastric muscles (DG, GM) in seven of eight, four of four, and three of three preparations, respectively. In all three cases, the spontaneous embryonic rhythm is restored after rinsing (recovery). In the case of GABA application on anterior ganglia, only preparations on which a single embryonic rhythm can be recorded in control conditions were included ( 3 of 10 preparations). The dissection of the anterior part of the preparation (a necessary step for selective application of GABA on anterior ganglia) can damage the modulatory system and precludes the expression of a single embryonic rhythm. Voltage levels (in millivolts) are indicated by arrowheads as follows: $A, G M,-40, P D,-50 ; B, D G,-65, P D,-35 ; C, D G,-70, L P,-58$.

application was restricted to the modulatory system (anterior ganglia), we observed a complete cessation of rhythmic activities $(n=3$ of 3$)$ (Fig. 3C).

These data show that both the embryonic STG network and its modulatory input system are sensitive to GABA, but that the application of exogenous GABA is not sufficient to elicit the expression of the three adult-like networks in embryonic STNS preparations.

\section{Effects of GABA deprivation in the adult STNS}

Bath application of GABA on the embryonic STNS did not mimic the effects of neurally released GABA in the STG, presumably because it cannot be specifically applied to the targets of the eight known modulatory neurons that will express GABA in the adult (Cournil et al., 1990). Therefore, the potent inhibitory effect of GABA found here and also reported in adult STNS may mask a possible induction of adult rhythms by GABA in the embryonic STNS. Hence, to overcome this issue, we used the opposite experimental strategy of preventing GABA synthesis within adult modulatory neurons projecting to the STG.

We used 3-MPA, a potent inhibitor of the GABA synthesis enzyme (glutamic acid decarboxylase), to inhibit the production of GABA within the adult neuromodulatory input system (for the specificity of 3-MPA, see Materials and Methods). Because all GABAergic modulatory neurons projecting to the STG have their soma in the OG and CoG, we performed bath application of $1 \mathrm{~mm}$ 3-MPA onto the adult rostral ganglia ( $\mathrm{OG}$ and $\mathrm{CoG}$ ) while recording simultaneously pyloric, gastric, and esophageal rhythms $(n=9)$. Because the esophageal network is built of neurons distributed across several ganglia, we monitored esophageal activities both in the STG (through intracellular recordings of IPSP cells, nonspiking cells receiving large IPSPs from other esophageal neurons) and in the anterior ganglia (through extracellular recordings of esophageal motor nerves).
One to $5 \mathrm{~h}$ after the administration of 3-MPA on rostral ganglia, we found that the normal adult pyloric, gastric, and esophageal rhythms recorded from STG neurons (Fig. 4A1,B1) began to be replaced by a single unified motor pattern reminiscent of embryonic stages (Fig. 4A2) ( $n=7$ of 9 , the remaining preparations being unaffected by $3-\mathrm{MPA}$ ), whereas esophageal motoneurons (located outside the STG) displayed a tonic activity (Fig. $4 A 2$, bottom trace). The mean cycle frequency of the unified rhythm expressed in the presence of 3-MPA [pyloric ( $0.76 \pm 0.06$ $\mathrm{Hz}$; mean $\pm \mathrm{SEM})$, gastric $(0.77 \pm 0.05 \mathrm{~Hz})$, and esophageal $(0.76 \pm 0.06 \mathrm{~Hz})$ neurons] (Fig. 4 B2) was significantly different from the mean cycle frequencies of control pyloric $(1.25 \pm 0.10$ $\mathrm{Hz})$, gastric $(0.16 \pm 0.01 \mathrm{~Hz})$, and esophageal $(0.47 \pm 0.06 \mathrm{~Hz})$ rhythms (Fig. 4 B1,B2) $(p<0.05$, one way ANOVA followed by Mann-Whitney test). The effect of 3-MPA was reversible after rinsing, because the cycle frequencies of all three rhythms returned to control adult values within 1 to $2 \mathrm{~h}$ after removing 3-MPA (Figs. $4 A 3, B 3)$ ( $n=2$ of 2 ; most preparations were not tested for rinsing as they were used for dye-coupling or GABA immunostaining experiments) (see below). In 13 additional preparations in which only pyloric and gastric activities were monitored, 3-MPA application to the anterior ganglia also induced the expression of a unified motor pattern in eight preparations, the remaining preparations being unaffected by 3-MPA. In total, a unified rhythm was induced by 3-MPA in 15 of 22 preparations. Finally, we checked whether 3-MPA induced a modification of neuron membrane potentials. No statistically significant difference could be found in the membrane potentials of recorded neurons between control and 3-MPA conditions (paired $t$ test, $p>0.05 ; n=35$ ).

To examine whether the effect of 3-MPA was caused by a reduction of GABA within the modulatory input system, we undertook GABA immunocytochemistry after 3-MPA application in 11 preparations [six expressing unified rhythmic activity (Fig. 
A1 Control

B1

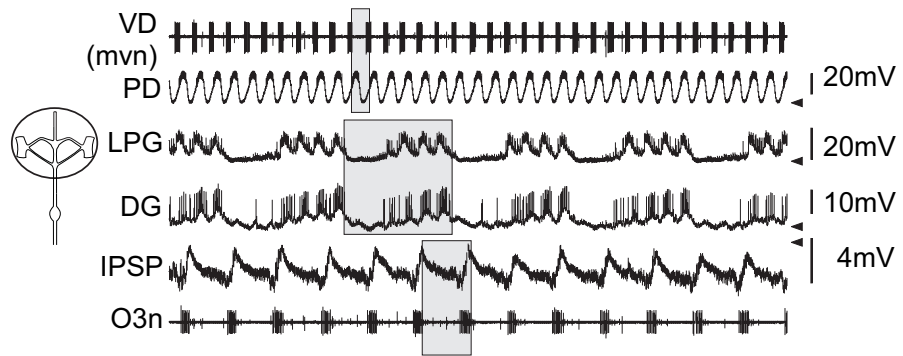

A2

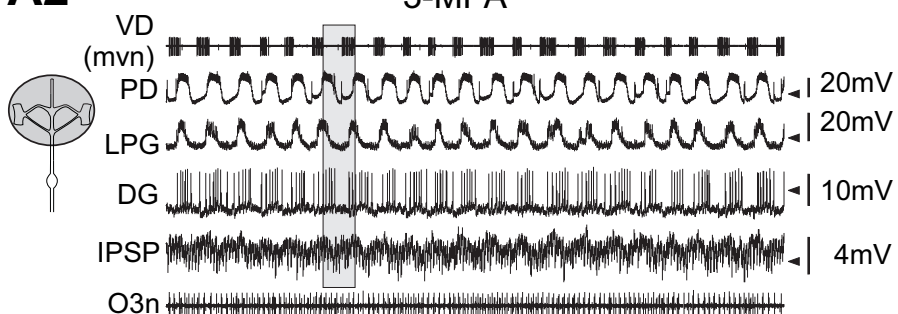

A3

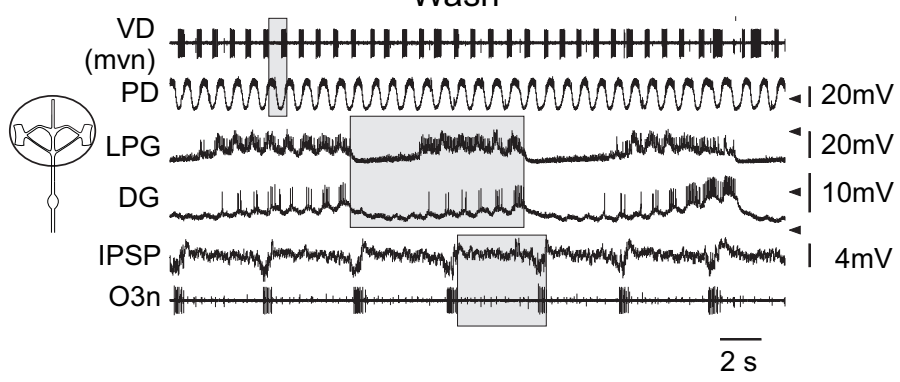

Wash

B3

Figure 4. Inhibition of GABA synthesis within adult anterior ganglia using 3-MPA induces the expression of a unified motor pattern within STG neurons. A1-A3, Electrophysiological recordings of the rhythmic activities produced by pyloric [ventral dilator (VD), PD], gastric (LPG, DG), and esophageal (IPSP, dilator esophageal) neurons in control conditions (A1), after $4 \mathrm{~h}$ of superfusion of $1 \mathrm{~mm}$ 3-MPA on the anterior ganglia (grayed area on the schematic drawing, A2), and after $1 \mathrm{~h}$ washing 3-MPA (A3). After 3-MPA treatment, a unified rhythmic motor pattern $(\boldsymbol{A 2})$ is produced in place of the three adult pyloric, gastric, and esophageal motor patterns $(\boldsymbol{A} \mathbf{1}, \boldsymbol{A} \mathbf{3})$. Gray boxes highlight the periods of the pyloric, gastric, esophageal $(\boldsymbol{A} \mathbf{1}, \boldsymbol{A} \mathbf{3})$ and unified $(\boldsymbol{A} \mathbf{2})$ rhythms. Note that the esophageal motor nerve does not display this unified rhythmic activity but instead fires tonically. PD, LPG, DG, and IPSP were recorded intracellularly whereas the activity of VD and dilator esophageal motoneuron was recorded extracellularly on the medial ventricular nerve (mvn) and esophageal nerve three (03n; large spikes on the extracellular recording), respectively. Arrowheads on the side of the traces indicate voltage levels for PD $(-60 \mathrm{mV}), \mathrm{LPG}(-60 \mathrm{mV}), \mathrm{DG}(-95 \mathrm{mV})$, and IPSP $(-75 \mathrm{mV})$. B1-B3, Quantification of the mean cycle frequency of pyloric (PD), gastric (DG or LPG), or esophageal (IPSP) STG neurons measured in seven experiments in control conditions (B1), after 3-MPA application (B2), and after washing 3-MPA (B3; $n=2)$. For each experiment, cycle frequencies were measured over a period of at least $1 \mathrm{~min}$. Error bars indicate SEM.

Finally, it should be noted that bath application of 3-MPA restricted to the STG alone did not alter the ongoing rhythmic activity of the preparations ( $n=3$ of 3 ) (Fig. 6A,B). Specifically, the intrinsic cycle frequency of the pyloric $(1.01 \pm 0.09 \mathrm{~Hz})$, gastric $(0.15 \pm 0.03 \mathrm{~Hz})$, and esophageal $(0.24 \pm 0.08 \mathrm{~Hz})$ rhythms was unaltered relative to control groups $(1.08 \pm 0.16 \mathrm{~Hz}$, $0.15 \pm 0.02 \mathrm{~Hz}$, and $0.23 \pm 0.12 \mathrm{~Hz}$, respectively; paired $t$ test, $p>0.05 ; n=3$ ). Additional immunocytochemical detection of GABA was performed in two of these preparations and revealed normal GABAergic staining both in the OG (Fig. $6 \mathrm{Cl}$, four stained somata) and the CoGs (Fig. 6C2), as well as a pattern of neuropilar staining clearly visible in the anterior part of the STG (Fig. 6C3). These experiments suggest that most of the GABA detected in the STG does not result from a local GABA synthesis within modulatory neurons terminals. It also demonstrates that 3-MPA does not seem to alter chemical synaptic transmission mediated by glutamate and acetylcholine, which are the two main neurotransmitters used by gastric and pyloric neurons in related species (Marder, 1974; Marder and Eisen, 1984), as the control rhythms are maintained under 3-MPA application on the STG.

Together, these results indicate that a reduction of GABA levels within the adult modulatory input system results in the appearance of a single unified motor pattern within the adult STG neurons. Several hypotheses may be suggested to explain the production of a unified rhythm after treatment with 3-MPA. First, the pyloric, gastric, and esophageal STG neurons may operate independently at similar cycle frequencies. Second, a shared input may drive the pyloric, gastric, and esophageal STG neurons on a common rhythm. Third, the expression of the unified rhythmic activity may result from a functional reconfiguration of the adult STG neurons

5A1) and five remaining unaffected by 3-MPA (Fig. 5B1)]. GABAergic immunoreactivity showed a marked decrease in preparations expressing a unified motor pattern after 3-MPA superfusion (compare Figs. $2 B$, adult micrographs, $5 A 2-A 4$ ). Indeed, in the OG, the mean number of stained somata $(1.33 \pm 0.6)$ was statistically lower in these preparations than in control adults $(3.46 \pm 0.21$, ANOVA followed by Dunn's test) (Fig. 5A2). Additionally, no $(n=2)$ or only rare $(n=4)$ GABAergic fibers and/or cell bodies were present in the CoGs (Fig. 5A3), and no neuropil or fibers were evident in the STG ( $n=3$ of 3 ) (Fig. 5A4). In contrast, the OG of 3-MPA-treated preparations expressing three distinct rhythms (Fig. 5B1) exhibited a mean number of stained somata $(2.6 \pm 0.6)$ that was not statistically different from control adult preparations (Fig. 5B2) and a marked staining persisted both in the CoGs (Fig. 5B3, fibers and somata) as well as in the STG neuropil ( $n=2$ of 2) (Fig. 5B4). into a single rhythmic motor network.

To rule out the possibility that the three independent networks operate at similar cycle frequencies, the phase relationships of each STG neuron involved in the generation of this unified motor pattern were analyzed over a maximum of 15 experiments using $\mathrm{PD}$ as the reference neuron (Fig. 7A). To achieve this analysis, the percentage of the total number of spikes occurring within a given phase was calculated for each motoneuron. For IPSP cells, that never express spiking activity, the mean voltage variation as a function of the phase was used. These results showed no phase drift in the phase of the discharge of each neuron, and the firing phase for each neuron was constant from one experiment to another, as indicated by the unimodal distribution of these phase-discharge curves (Fig. 7A). Therefore, the unified motor pattern expressed in the presence of 3-MPA does not result from independent networks operating at very similar cycle fre- 


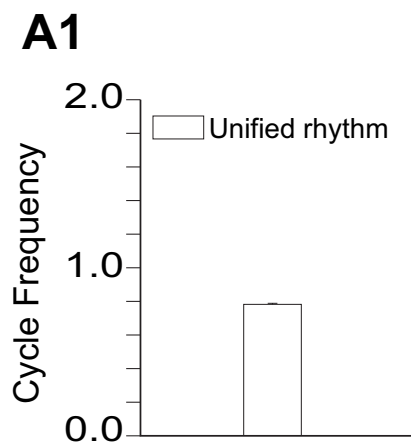

\section{B1}
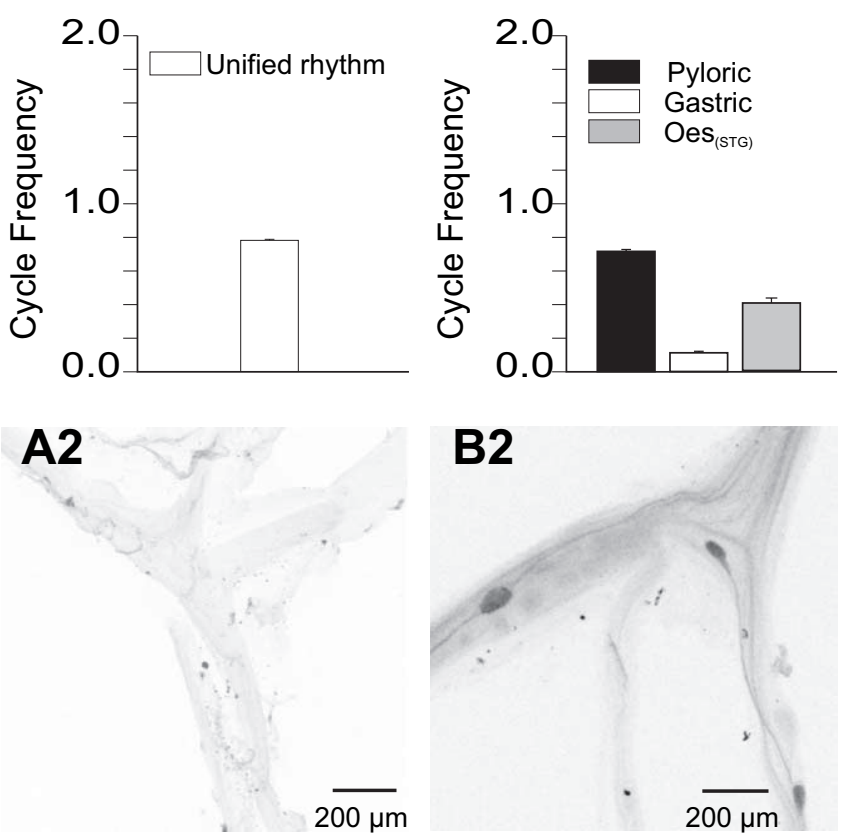

A3
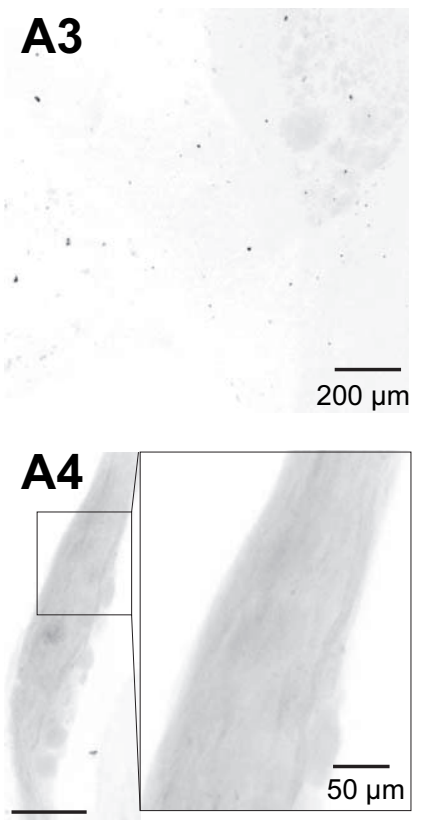

$\overline{200 \mu \mathrm{m}}$
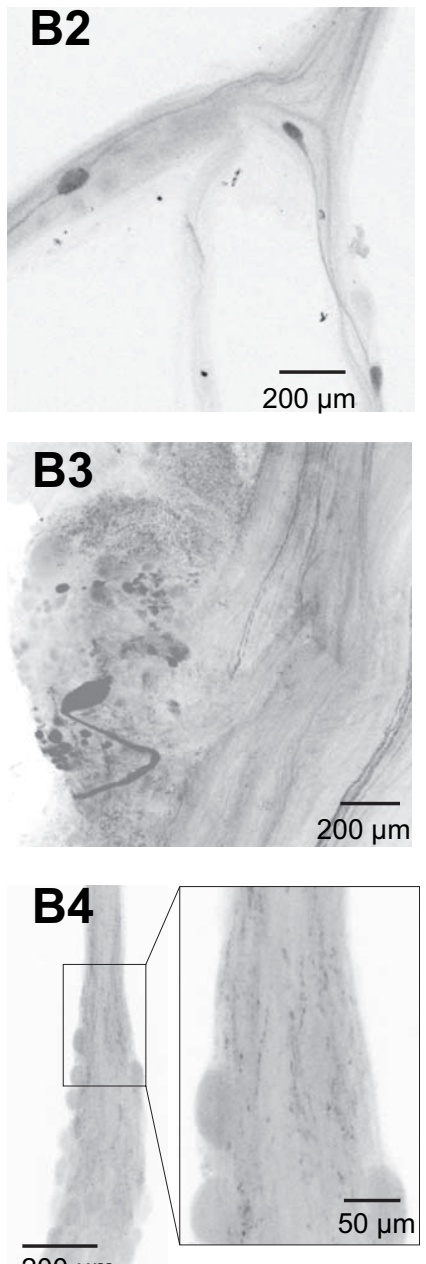

$\overline{200 \mu \mathrm{m}}$
Figure 5. 3-MPA application on anterior neuromodulatory ganglia induces a reduction of GABA within neuromodulatory inputs projecting to the STG in preparations expressing the unified motor pattern. A1-A4, Preparations expressing a unified rhythmic motor pattern after 3-MPA application $(\boldsymbol{A 1} ; n=6)$ treated for immunocytochemical detection of GABA reveal reduced staining in the $O G(\boldsymbol{A} \mathbf{2}$, example of a preparation in which no GABAergic neuron could be detected) and in the $\operatorname{COG}(\boldsymbol{A} 3)$ and the absence of neuropil in STG ( $n=3$ of 3$)(\boldsymbol{A 4}$, box on the right is an enlargement of a part of the STG). $\boldsymbol{B 1} \mathbf{B}$ 4, Preparations expressing three distinct rhythms after $>4 \mathrm{~h}$ 3-MPA treatment (B1, quantification of pyloric, gastric, and esophageal cycle frequencies) ( $n=5$ ) treated for immunocytochemical detection of GABA reveal the presence of several stained soma in the $0 G$ (B2, example of a preparation in which 4 GABAergic neurons could be detected, an additional GABAergic soma being located out of the frame of this micrograph), numerous stained cell bodies as well as fibers in the $\mathrm{CoG}(\boldsymbol{B} 3)$, and neuropilar staining in the STG (B4, box on the right is an enlargement of a part of the STG). Note that $\boldsymbol{A 2}-\boldsymbol{A} \mathbf{4}$ as well as $\boldsymbol{B} \mathbf{2} \boldsymbol{B} \mathbf{4}$ are from distinct preparations. Error bars indicate SEM. quencies. In addition, we performed resetting experiments (Fig. $7 B)$ in which the injection of brief hyperpolarizing current pulses in the soma of a pyloric (Fig. $7 B 1)(n=7$ of 7 preparations) or gastric (Fig. 7B2) ( $n=4$ of 4 preparations) neuron was able to reset the ongoing activity of all monitored neurons (pyloric, gastric, and esophageal STG neurons). For example, a brief hyperpolarization of PD (Fig. 7B1) resulted in a phase delay of all monitored neurons (compare the expected firing time indicated by arrowheads with the actual discharge of each neuron). Consequently, the expression of the unified rhythm does not result from the presence of a common phasic input that would drive the three pyloric, gastric, and esophageal rhythms at the same frequency. All gastric, pyloric, and esophageal STG neurons are phase-locked in the unified motor pattern, and brief current injection in at least some of these neurons [PD, $n=7$; lateral posterior gastric (LPG), $n=4$; dorsal gastric (DG), $n=1$ ] was able to reset the ongoing activity of all monitored neurons. Hence, we conclude that with the unified motor pattern obtained under 3-MPA application, all STG neurons are members of a single motor network that result from the functional reconfiguration of the adult pyloric, gastric, and esophageal networks into a single network.

\section{Comparison of the adult 3-MPA induced rhythm and the spontaneous embryonic rhythm}

The reduction of GABA levels within the adult modulatory neurons results in a unified rhythm in adult STG neurons. In the embryonic preparations, where GABA is similarly not expressed by modulatory inputs, STG neurons are also organized into a single functional network (Casasnovas and Meyrand, 1995) (Fig. 2). We therefore addressed whether these unified rhythms in 3-MPA-treated adults and embryonic preparations are generated by the same functional STG network by comparing the two unified motor patterns expressed in the adult and in the embryo.

We focused our comparison on pyloric and gastric neurons. In the embryo, the activity of IPSP cells cannot be monitored reliably. Comparisons of the main features of the 3-MPA induced unified rhythm recorded from adult STG neurons and nerves (Fig. 8A1) with the spontaneous embryonic rhythm recorded from stomodeum muscles (Fig. 8A2) revealed striking similarities. First, we found that the two networks operate at the same frequency. The mean firing frequency of the unified adult 3-MPA rhythm $(0.87 \pm 0.07 \mathrm{~Hz} ; n=13)$ was not different to the mean firing frequency of the spontaneous embryonic rhythm $(0.79 \pm$ $0.07 \mathrm{~Hz} ; n=9$, Mann-Whitney test). Second, the overall rhythmic patterns generated by the adult network under 3-MPA and the embryonic network were very similar (Fig. 8A,B). A quantification of the phase of discharge of each neuron that could be recorded both in adult and in embryo over several experiments (up to 10 in the embryo) revealed that the sequence of activation looks very similar in both cases $[\mathrm{PD}, \mathrm{DG}$, lateral gastric (LG), lateral pyloric (LP), gastric mill (GM), pyloric motoneuron (PY)] (Fig. 8, compare B1, B2). For neurons for which this can be assessed (PD, DG, LP, GM; $n>3$ ), no statistical difference was found in the mean phase of discharge between the embryo and adult 3-MPA rhythm (Watson's $U^{2}$ ), although differences could be observed in the distribution of events throughout one cycle (compare for example adult and embryonic DG).

These studies demonstrated that embryonic and adult 3-MPA rhythms are both generated by a single functional network (Casasnovas and Meyrand, 1995; present study) that displays identical operating frequencies and identical sequences of muscle 

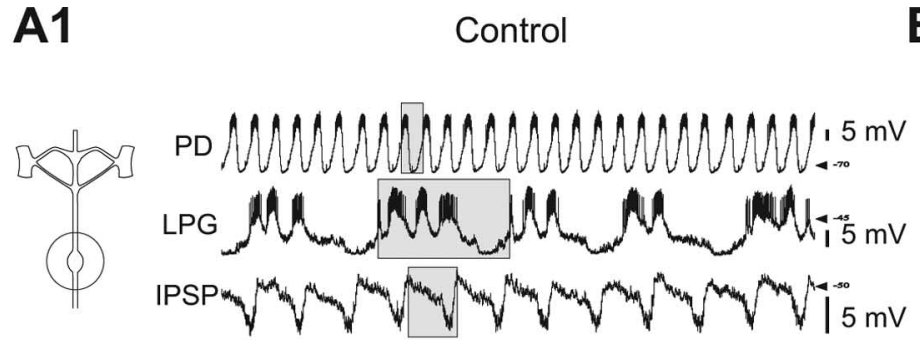

A2
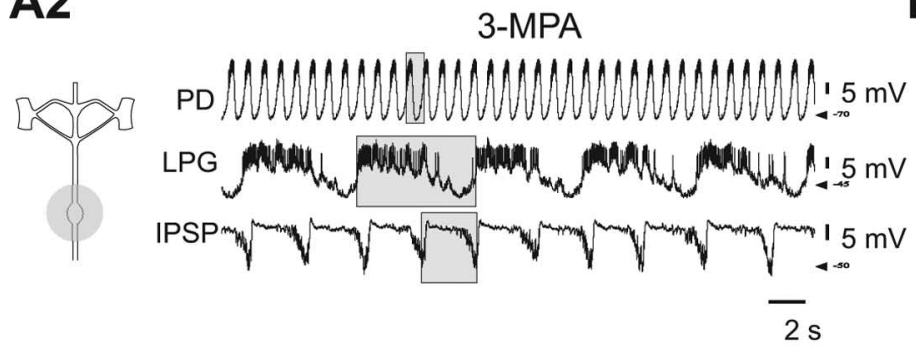

C1

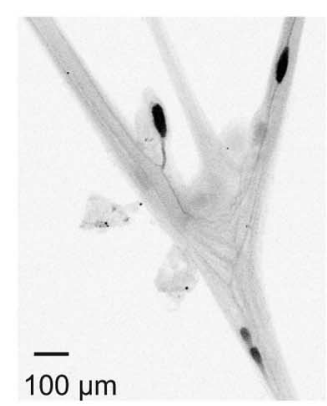

C2

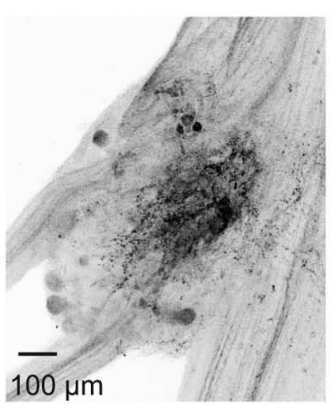

C3

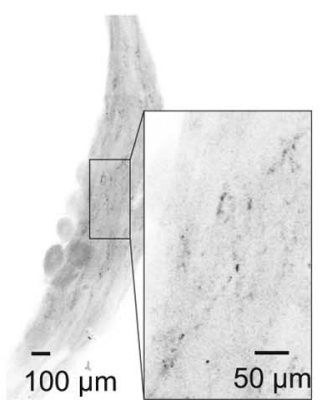

Figure 6. 3-MPA application on the STG does not alter the rhythmic motor patterns expressed by STG neurons. $\boldsymbol{A} \mathbf{1}, \boldsymbol{A 2}$ Intracellular electrophysiological recordings of the rhythmic activities produced by pyloric (PD), gastric (LPG), and esophageal (IPSP) neurons in control conditions (A1) and after $5 \mathrm{~h}$ of superfusion of $1 \mathrm{~mm} 3$-MPA on the STG $(\boldsymbol{A 2}$, grayed area on the schematic drawing). Gray boxes on recordings highlight the periods of the pyloric, gastric, and esophageal rhythms. Arrowheads on the side of the traces indicate voltage levels for PD $(-70 \mathrm{mV})$, LPG $(-45 \mathrm{mV})$, and IPSP $(-50 \mathrm{mV}) . \mathbf{B 1}, \boldsymbol{B}$, Quantification of the mean cycle frequency of pyloric (PD), gastric (LPG), or esophageal (IPSP) STG neurons measured in three experiments in control conditions (B1) and after 3-MPA application on the STG (B2). For each experiment, cycle frequencies were measured over a period of at least $1 \mathrm{~min}$. C1-C3, Immunocytochemical detection of GABA in preparations incubated in the presence of 3-MPA on the STG reveals the presence of four GABAergic somata in the $0 G(C 1)$, numerous fibers and somata in the $C_{0} G s$ (C2), and faint neuropilar staining in the anterior part of the STG $(\mathbf{C} 3$, see high-magnification insert of neuropil staining in box). Error bars indicate SEM.

activation. Therefore, our data demonstrate that adult networks retain the ability to express embryonic-like patterns depending on their modulatory environment.

Dye-coupling in the presence or absence of GABA in the adult and in the embryo

Modeling studies have raised the possibility that the unification of distinct motor pattern generating networks into one may result from an increase in the extent and strength of electrical coupling (Bem et al., 2002). Demonstrating an alteration in the strength of electrical coupling would require a total blockade of the modulatory system as well as the chemical synaptic transmission within the STG, which would prevent the expression of the unified rhythm both in the adult and in the embryo. We therefore used neurobiotin, a low molecular weight tracer known to cross gap junctions, as a tool to investigate the extent of electrical coupling during the expression of the unified rhythm. We choose to inject one of the two PD neurons as these neurons are known to be electrically coupled to several other STG neurons. PD neurons,
B1

B2

identified in the adult and in the embryo using conventional electrophysiological techniques [extracellular spike recorded with constant latency on PD motor nerve in the adult (Fig. 9A1); excitatory junction potential recorded with constant latency on PD muscle in the embryo (Fig. 9A2)], were injected with neurobiotin. After histochemical processing, this procedure allows the visualization of the population of neurons dye-coupled to the injected PD neuron.

We found that the number of neurons dye-coupled to a PD neuron was significantly increased in adult preparations expressing the 3-MPA unified rhythm compared with control preparations (Fig. $9 B 1, B 2, C)$. The PD cells of control adults expressing typical pyloric, gastric and esophageal rhythms are dye-coupled to an average of seven to eight neurons (7.6 \pm 0.68 , ranging from 4 to $11 ; n=10$ ) (Fig. $9 B 1, C)$ whereas each PD neuron in 3-MPA-treated adults was coupled to an average of 20 neurons $(20.5 \pm 2.14$, ranging from 9 to $26 ; n=7$ ) (Fig. 9B2,C). In the embryo, we found that an average of 19-20 neurons (19.4 \pm 2.15 , ranging from 13 to $28 ; n=5$ ) (Fig. 9B4,C) dye-coupled to a PD neuron. Bath application of $10^{-3}$ M GABA to the embryo preparation significantly reduced the number of dyecoupled cells so that only four to five neurons were dye-coupled to each PD neuron $(4.25 \pm 1.18$, ranging from 1 to $6 ; n=4)$ (Fig. 9B3,C). Hence, dye-coupling is identical in embryonic and 3-MPA-treated adult preparations and much higher than that observed in control adult and GABAtreated embryonic preparations.

\section{GABA directly decreases electrical and} dye-coupling in the adult STG

Our data suggest that, in the absence of GABA within the modulatory input neurons, increased electrical coupling within the target networks result in the expression of a single rhythm whereas, in the presence of GABA, reduced electrical coupling induces the expression of three distinct rhythms. We therefore asked whether GABA was able to directly regulate the extent and strength of electrical coupling within the adult STG. To address this issue, the modulatory input system was pharmacologically blocked using $10^{-6} \mathrm{M}$ TTX to prevent an indirect action of bath-applied GABA through the modulatory system. Chemical inhibitory synapses mediated by chloride channels in the STG were blocked using $10^{-6} \mathrm{M}$ PTX. The effect of bath application of $10^{-3} \mathrm{M}$ GABA was then tested on the extent and strength of electrical coupling. First, as above, we used neurobiotin injection within a PD neuron to characterize the effect of GABA on the extent of electrical coupling. We found that the number of STG neurons dye-coupled to one adult PD was significantly ( $p<0.001, t$ test) reduced after bath application of $10^{-3}$ M GABA ( $3 \pm 0.44$, ranging from two to four; $n=5$ ) (Fig. $10 A 2, A 3)$ compared with control (adult TTX/PTX bathed prep- 
arations, $6 \pm 0.75$, ranging from four to nine; $n=7$ ) (Fig. 10A1). Second, by making simultaneous dual electrodes intracellular recordings from two PDs (Fig. $10 \mathrm{~B}$, voltage traces), we measured the coupling coefficient between the two PDs (known to be electrically coupled in the adult) in control conditions and after bath application of GABA $(n=4)$ (see Materials and Methods). We found that the coupling coefficient was decreased (by $>50 \%$ ) in the presence of GABA compared with control $(p<0.0001$, one way ANOVA followed by Tukey's test) (Fig. 9D). This effect was not observed until 10 min after GABA application and was reversible with rinsing $(p<0.0001)$, although a full return to control values was never obtained (after up to $2 \mathrm{~h}$ rinsing). This decrease in coupling coefficient was not attributable to a decrease in the input resistance of the PD neurons, as no statistically significant effect of GABA was found on the input resistance of the neuron (Fig. 10C). We conclude that GABA directly induces a decrease in the extent and strength of electrical coupling in the adult STG networks.

\section{Discussion}

Neuromodulation and the development of neural networks

We found that expression of GABA within modulatory inputs of the STNS after metamorphosis coincides with the expression of adult STG networks. However, GABA acquisition is not a sharp phenomenon as the number of GABAergic OG somata is more variable in LIV than in adult and only some LIV and juvenile preparations showed GABAergic neuropil staining in the STG. This progressive acquisition of GABA is not surprising as, although the metamorphic moult occurs between stage III and IV, some ecological, behavioral, and physiological changes, including changes in the diet, are only accomplished by the end of stage IV or during stage V (juveniles) (Charmantier et al., 1991). Adult-like motor patterns are expressed at the onset of stage IV, although they remain different from adult patterns (Casasnovas and Meyrand, 1995). Therefore, the gradual acquisition of GABA could account for the progressive emergence of adult rhythms after metamorphosis.

Our data further suggest that GABA acquisition within specific modulatory neurons triggers the expression of adult networks in the developing STNS. Indeed, although GABA application to the embryonic STNS did not induce the expression of an adult-like network, removing GABA from adult anterior ganglia induces the expression of an embryonic-like network. Several reasons may account for the inability of exogenously applied GABA to elicit adult-like motor patterns in embryo. First, GABA application is likely to influence both synaptic and extrasynaptic receptors whereas only synaptic receptors are likely to be influenced by GABA released from the four known pairs of GABAergic neurons (Cournil et al., 1990). Second, the cessation of
B1

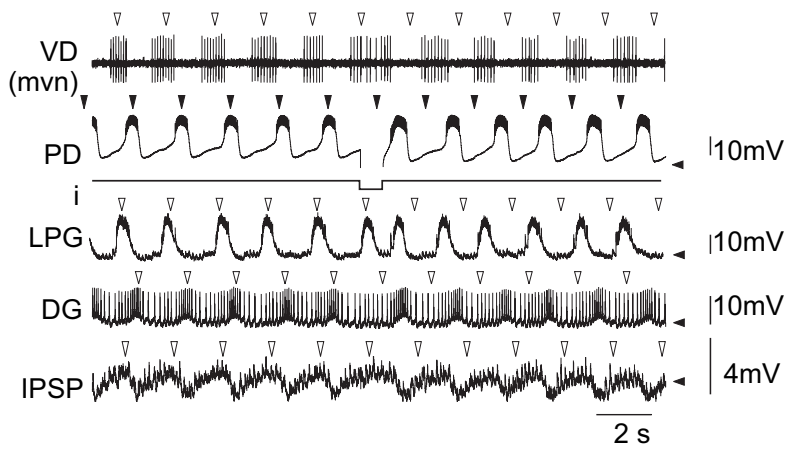

LPG $(n=14)$

DG B2 $(n=11)$

LG $(n=2)$ MG $(n=1)$ GM $(n=6)$ IPSP $(n=7)$

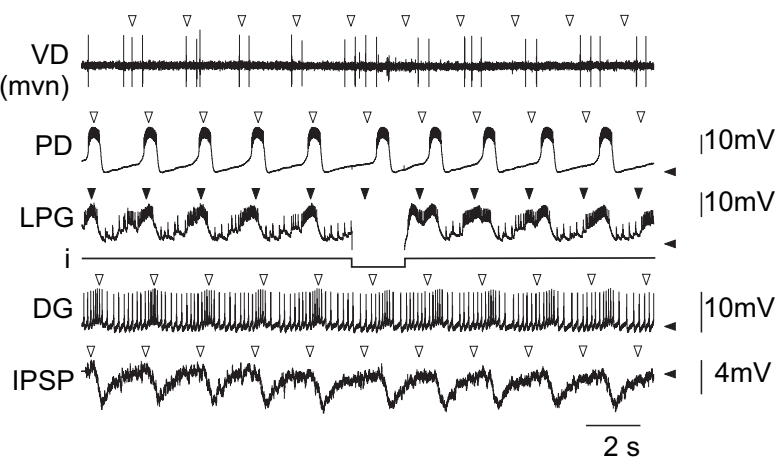

Figure 7. The unified activity produced by adult STG neurons under 3-MPA is generated by a single network. $A$, Distribution of the onset of the first spike of PD neuron). Measurements were performed over 1-2 $\mathrm{min}$ (at least 35 cycles in each preparation). The number of preparations used for each neuron is indicated in parentheses. B1, B2, Brief (500-1500 ms) injections of hyperpolarfor ventral dilator (VD)] or intracellular recordings expected firing time for each neuron in the absence of experimental perturbation. Arrowheads on the side of the traces indicate voltage levels for PD $(-72 \mathrm{mV}), \mathrm{LPG}(-53 \mathrm{mV}), \mathrm{DG}(-45 \mathrm{mV})$, and IPSP $(-35 \mathrm{mV})$. IC, Inferior cardiac.

embryonic-like activity after GABA superfusion could be caused by a widespread and massive inhibition of the modulatory system as well as the target networks. This potential impact on the modulatory system is a possibility because picrotoxin does not block all of the fast inhibitory actions of GABA in arthropods (Albert et al., 1986; Swensen et al., 2000). Third, it is possible that the ability of exogenous GABA to elicit adult-like motor patterns cannot be obtained before the acquisition of GABA within the modulatory input system. In Xenopus tadpoles, when 5-HT projections have only reached the rostral spinal cord, application of 5-HT mimics larval swimming activity in rostral but not caudal ventral roots (Sillar et al., 1992). However, this hypothesis seems unlikely in our preparation as we show that GABA is able to induce a decrease in gap-junctional coupling in the embryo, long before the acquisition of GABA immunoreactivity within the neuromodulatory system that innervates the STG.

\section{GABAergic modulation of gap junctional coupling}

We report that GABA reduces the extent and strength of electrical coupling (see also Piccolino et al., 1982; Shinohara et al., 2000). Surprisingly, we found no effect of GABA on the input resistance 
A1

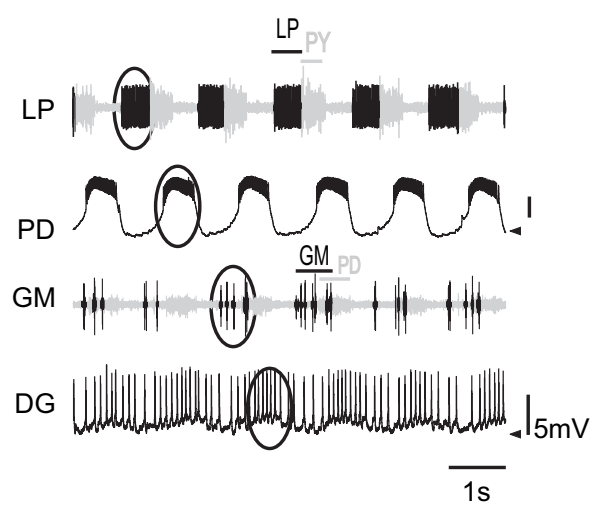

B1
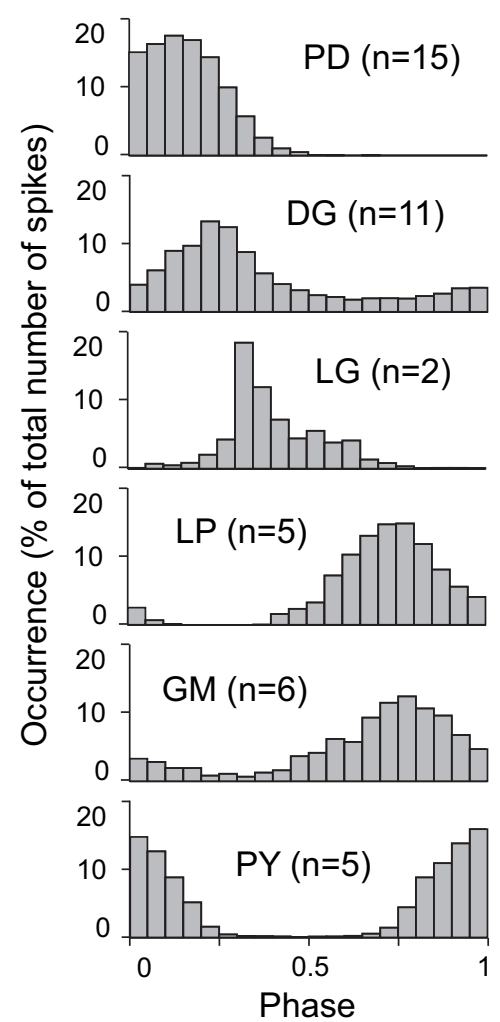

A2
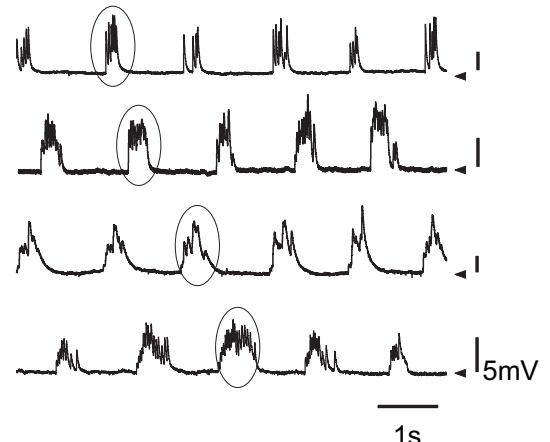

B2
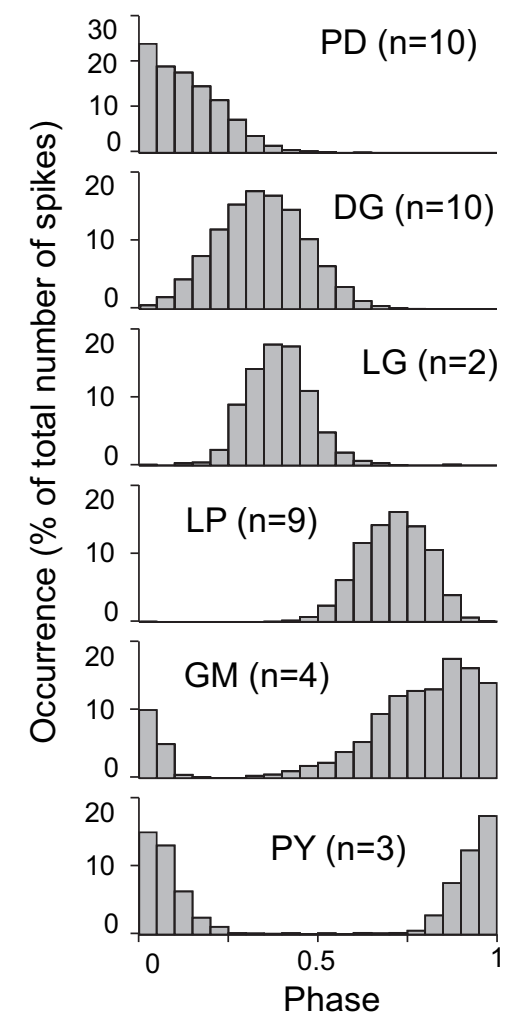

Figure 8. The adult 3-MPA and the embryonic rhythms display similar phenotype of rhythmic patterned activities. $\mathbf{A 1}, \mathbf{A 2}$, Recordings of the unified rhythm in the adult 3-MPA treated (A1) and in the embryo (A2). Circles highlight the successive discharges of the recorded neurons in two consecutive cycles. In adult (A1) PD and DG were recorded intracellularly from neuron soma whereas $L P$ and $G M$ were recorded extracellularly from the motor nerves. Note that for both extracellular recordings ( $L P$ and $\mathrm{GM}$ ), discharges of other neurons are visible on the nerves (PY and PD, respectively). The discharges of the neuron of interest are represented in black. In the embryo (A2), all traces are intracellular recordings of the target muscles of the same neuron as in $\boldsymbol{A} \mathbf{1}$. Voltage levels (in millivolts) are indicated by arrowheads as follows: $A 1, P D,-60, D G,-50 ; A 2, L P,-80, P D,-75, G M,-70$, $D G,-60 . B 1, B 2$, Distribution of firing as a function of the phase in the adult (B1) and the embryonic (B2) single rhythm. The period of $\mathrm{PD}$ neuron was taken as a reference for phase computations ( $t=0$ for first spike in PD). Vertical bars represent the percentage of the total number of spikes that occurred within a given phase. Measurements were performed over 1-2 min (at least 35 cycles in each preparation). The number of preparations used for each neuron is indicated in parenthesis. Note that the overall sequence of activation is identical in both 3-MPA treated adult (B1) and in the embryo (B2).

of the studied neuron. At least two hypotheses may account for this surprising result. First, the increase in input resistance mediated by gap junction closure may have been compensated by an opening of GABA sensitive channels that cannot be fully blocked by the pharmacological agents we used (Swensen et al., 2000). Second, as potential sites for electrical synapses between the two PDs are sparse and distant from the soma (E. Ducret, personal communication), we may have been unable to detect a change in the total input resistance measured at the soma level.

In the crab STG, GABA evokes both depolarizing and hyperpolarizing responses (Swensen et al., 2000). In crayfish skeletal muscles (Kaila and Voipio, 1987) as well as hippocampal pyramidal cells (Perkins and Wong, 1996), the depolarizing response to GABA is predominantly carried by $\mathrm{HCO}_{3}^{-}$that leads to a dramatic fall in intracellular $\mathrm{pH}$. As in other species, intracellular acidification produces electrical uncoupling at crayfish axons (Peracchia, 1990). Therefore, GABA, by inducing an efflux of $\mathrm{HCO}_{3}^{-}$, may in turn decrease electrical coupling. However, thus far, there is no evidence that STG GABAergic ionotropic receptors are permeant to $\mathrm{HCO}_{3}^{-}$. Given the long-term effects of GABA on electrical coupling in our preparation, a metabotropic GABA action cannot be ruled out. Although $\mathrm{GABA}_{\mathrm{B}}-$ like receptors have been reported at the lobster neuromuscular synapse (Miwa et al., 1990), no information regarding the presence of $\mathrm{GABA}_{\mathrm{B}}$-like receptors within the lobster STG is available.

Finally, although our experiments suggest a direct action of GABA on electrical coupling, GABA may also act at the CoGs level by modifying the activity of other modulatory neurons projecting to the STG, or even through presynaptic inhibition in the STG. We were unable to determine the relative importance of these two pathways. Indeed, we could not independently deplete GABA in the STG (Fig. 6) or in the anterior ganglia (Fig. 5).

\section{Network reconfiguration as a developmental mechanism}

In the embryo, esophageal, gastric, and pyloric neurons are all involved in the unified rhythm. In contrast, our results show that within the adult esophageal network, IPSP cells that are located in the STG are involved in the single functional network observed under 3-MPA whereas esophageal motoneurons located in the OG and CoG display tonic activity. Two hypotheses may account for this partial recruitment of esophageal network members. First, GABA deprivation within the adult anterior ganglia does not fully mimic the embryonic state as GABA is already present within the embryonic CoGs and may be necessary for the expression of rhythmic activity in esophageal motoneurons. Second, embryonic STG neurons may influence 


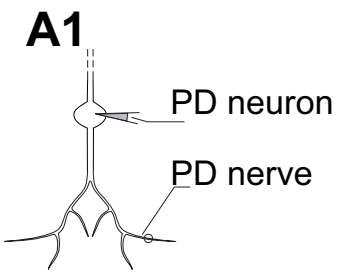

Adult
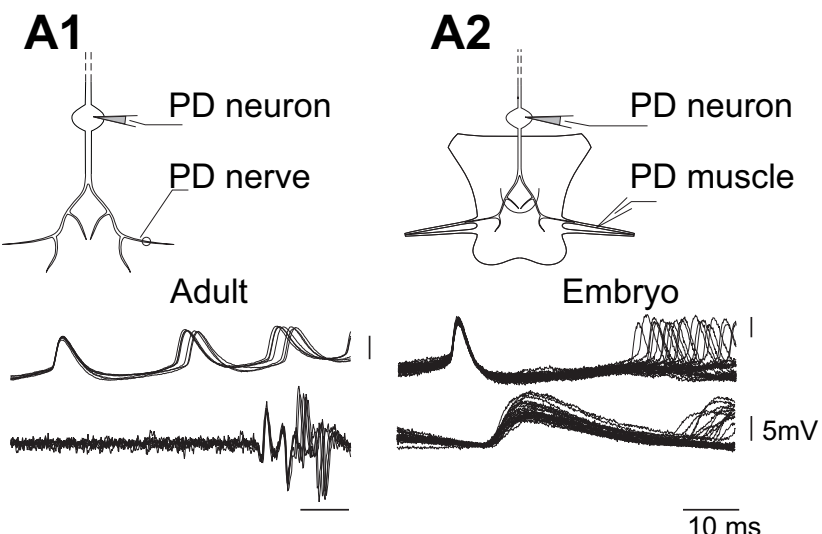

Adult

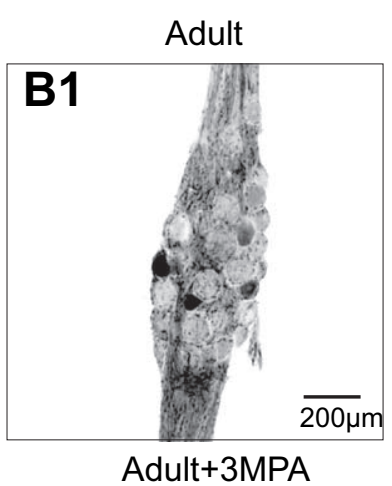

Embryo+GABA
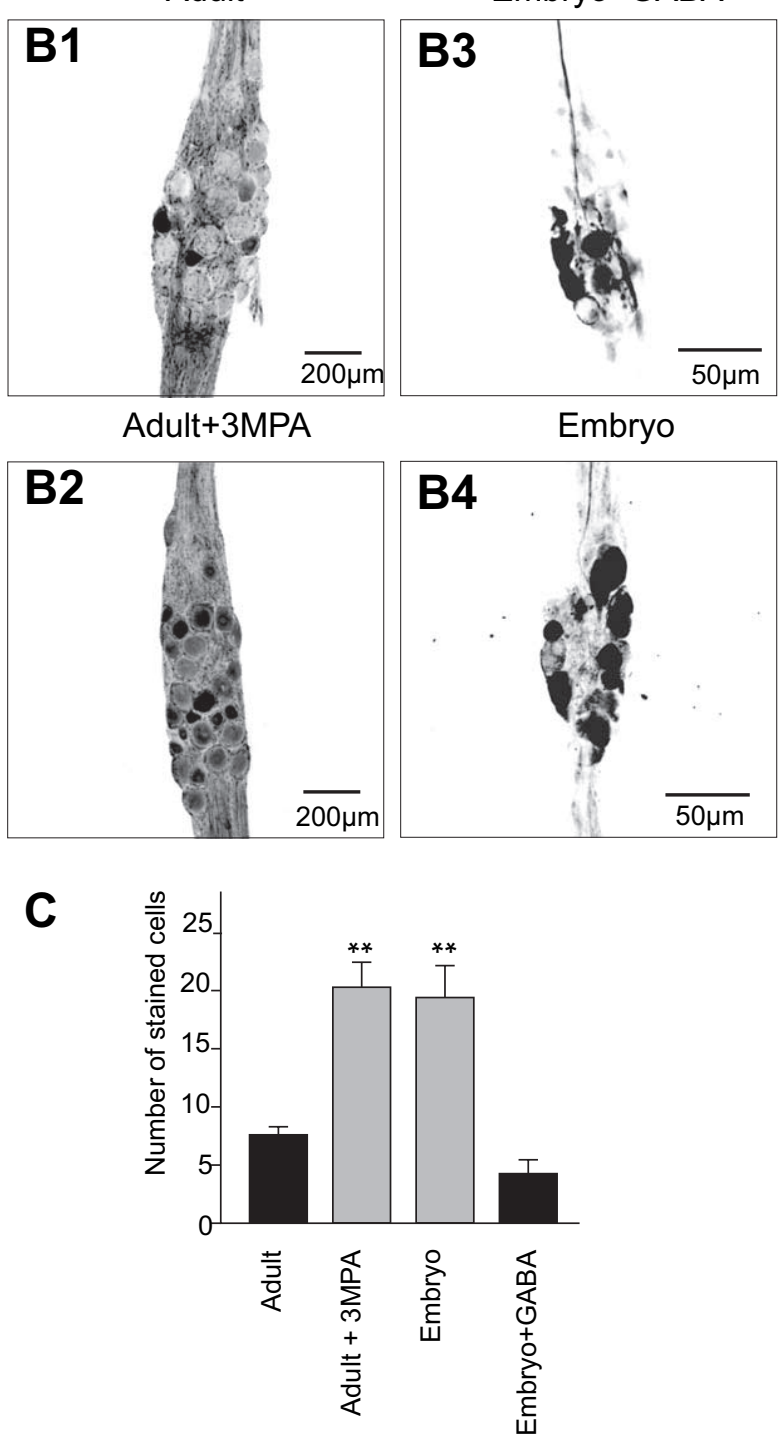

Figure 9. The expression of the unified rhythmic activity both in the adult (3-MPA treated) and in the embryo is associated with an increase in the extent of dye-coupling. A1, A2, PD neurons were identified in the adult $(\boldsymbol{A} \mathbf{1})$ on the basis of a constant latency extracellular action potential recorded on PD motor nerve $(\boldsymbol{A} \mathbf{1}$, bottom traces, 7 superimposed traces) after each intracellularly recorded spike (A1, top traces), and on the basis of a constant latency excitatory junction potential recorded in the muscle in the embryo $(\boldsymbol{A} 2$, bottom traces for muscle and top traces for neuron, 26 superimposed traces). $\boldsymbol{B 1 - B 4}$, Neurobiotin injections of a PD neuron in control adult (B1), in the 3-MPA-treated adult (B2), and in the control embryonic (B4) and in the GABA-treated embryonic preparations $(B 3)$. All images are maximal projections of a full confocal stack of 49-67 images, each $2.5 \mu \mathrm{m}$ thick. C, Quantification of the number of stained esophageal motoneurons because of short electrotonic distances between them, whereas in the adult, the long electrotonic distance between the STG and the anterior ganglia may preclude such influence. Despite the partial recruitment of esophageal neurons, our data suggest that adult networks retain the ability to express an embryonic-like phenotype (see also Sullivan et al., 2002) and that they can be reconfigured into a single functional network generating an embryonic-like motor pattern.

We demonstrated previously that the single embryonic STG network can be reconfigured into two distinct functional networks expressing adult-like motor patterns (Le Feuvre et al., 1999). Together, these results indicate that the expression of adult networks after metamorphosis results from a reversible reconfiguration of a single premetamorphic network into multiple adult networks. Network reconfiguration is known to underlie adult adaptive plasticity (Meyrand et al., 1991; Combes et al., 1999; Lieske et al., 2000; Faumont et al., 2005). We propose here that reversible network reconfiguration may also constitute a key developmental mechanism. This contrasts with the current view of network ontogeny which is classically thought to involve longterm irreversible phenomena such as progressive acquisition of adult ion channels (Spitzer et al., 2002), alterations in neurotransmitters (Allain et al., 2005), or synaptic properties (Ben-Ari, 2002). Our data provide evidence that the switch from embryonic network to adult networks can be reversed. Therefore, one can ask what is the role of progressive ontogenetic changes in cellular and synaptic properties that have been observed in several preparations.

We show that, at our level of analysis, the unified rhythm expressed in the embryo and in GABA-deprived adult STG neurons are very similar, and therefore may be generated by the same functional network. However, recent work has emphasized that virtually undistinguishable motor patterns can nevertheless be produced by different ion channels and synaptic combinations within a given network (Prinz et al., 2004). Therefore, we cannot exclude that the combination of expressed channels is different in embryonic and adult STG neurons as suggested in Homarus americanus (Richards et al., 1999; Richards and Marder, 2000), although both can express either embryonic- or adult-like motor patterns. Finally, the presence of gap junctions could allow the sharing of conductances across several cells, one cell being strongly affected by the currents expressed by electrically coupled cells. According to such an hypothesis, altering the extent and strength of electrical coupling may constitute a powerful mechanism to reconfigure network activities.

\section{Gap junctions and developmental plasticity}

Our data suggest that GABA-induced gap junction closing may be the main mechanism through which the embryonic network reconfigures into the multiple adult networks. Widespread gapjunctional coupling is commonly observed in developing nervous systems (Kandler and Katz, 1995) and considerable previous work has emphasized the metabolic role of such coupling (Kandler, 1997). Widespread electrical coupling may also allow the synchronization of large neuronal assemblies as well as provide

$\leftarrow$

soma in the control adult $(n=10)$, the 3-MPA-treated adults $(n=7)$, the control embryos $(n=5)$, and the GABA-treated embryos $(n=4)$ reveals that the number of stained soma is significantly higher in the 3-MPA-treated adults and in the embryos compared with control adults as well as compared with GABA-treated embryos $\left({ }^{* *} p<0.001\right.$, one way ANOVA followed by Tukey's). Error bars indicate SEM. 
A1

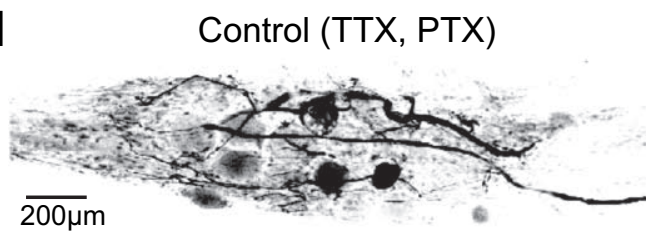

A2

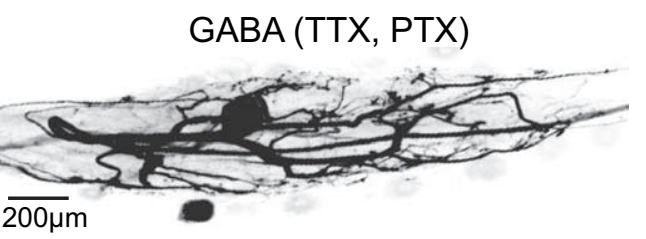

B

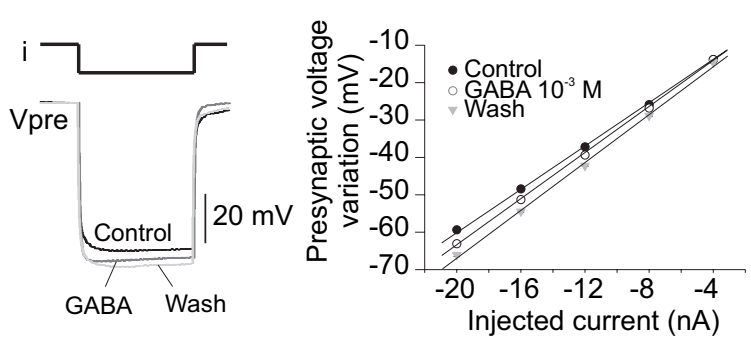

D1

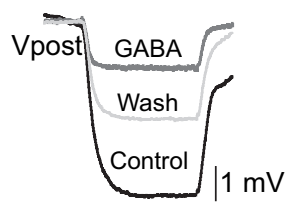

$\overline{0}, 1 \mathrm{~s}$

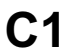

A3

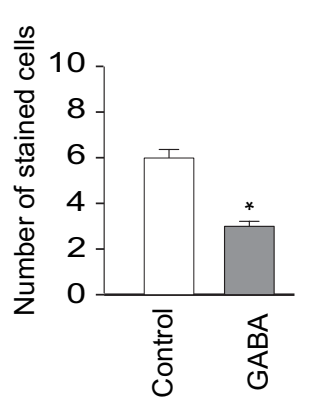

C2
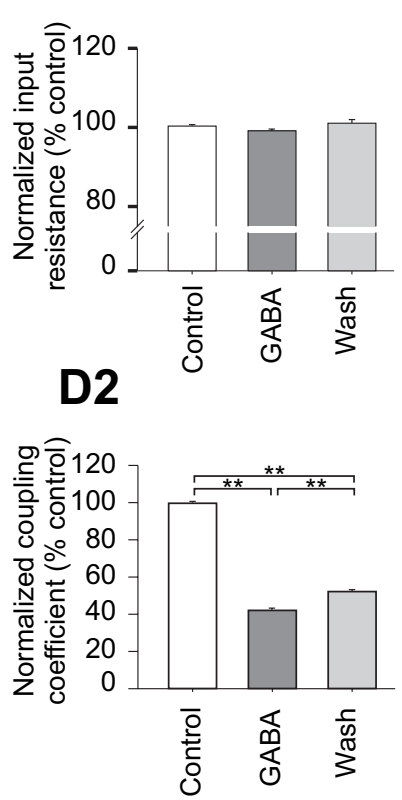

Figure 10. GABA directly modulates the extent and strength of electrical coupling in the adult system. $\mathbf{A 1}-\mathbf{A} \mathbf{3}$, Neurobiotin injection into the soma of an identified adult PD neuron in control conditions $\left(10^{-7} \mathrm{M} \mathrm{TTX}\right.$ and $10^{-6} \mathrm{M} \mathrm{PTX}$; A1) and after bath application of $10^{-3} \mathrm{M}$ GABA (TTX, PTX; A2). The number of neurons dye-coupled to PD is significantly ( ${ }^{*} p<0.01, t$ test) reduced after bath application of $10^{-3} \mathrm{M} \mathrm{GABA}(n=5)$ compared with control preparations treated with $10^{-7} \mathrm{M}$ TTX and $10^{-6} \mathrm{M}$ PTX $(n=7 ; A 3)$. Images are maximal projections of confocal stacks of $37-44$ images, each $2.5 \mu \mathrm{m}$ thick. $\boldsymbol{B}$, Voltage deflection induced by the injection of a brief current pulse $(0.5 \mathrm{~s},-20 \mathrm{nA})$ into presynaptic (Vpre; top) and postsynaptic (Vpost; bottom) PDs in control conditions ( $10^{-7} \mathrm{M} \mathrm{TTX}$ and $10^{-6} \mathrm{M}$ PTX), after $30 \mathrm{~min}$ of $10^{-3} \mathrm{M} \mathrm{GABA}$ and after $1 \mathrm{~h}$ wash of GABA. Because GABA induces a statistically significant hyperpolarization $(-7.81 \pm 1.56 \mathrm{mV}$, paired $t$ test) of PD neurons in the experimental conditions used (TTX, PTX), the traces corresponding to control, GABA, and wash were overlaid for clarity. Membrane potentials were as follows: control, $-63 \mathrm{mV}$ (Vpre) and $-57 \mathrm{mV}$ (Vpost); GABA, $-71.5 \mathrm{mV}$ (Vpre) and $-56 \mathrm{mV}$ (Vpost); wash, $-68 \mathrm{mV}$ (Vpre) and -55.3 $\mathrm{mV}$ (Vpost). C1, C2, The input resistance of the presynaptic PD neuron in all conditions can be measured as the slope of the curve showing voltage variation as a function of the injected current (C1). Quantification of the normalized input resistance (C2) over four experiments reveals that GABA has no effect on the input resistance of the cell. Only the points corresponding to negative current pulses are represented in this figure (linear part of the input/output curve). D1, D2, The coupling coefficient of the two PDs can be measured as the slope of the curve showing the variation of postsynaptic voltage as a function of presynaptic voltage (D1). Quantification of the normalized coupling coefficient (D2) over four experiments shows that GABA induces a marked decrease in the coupling coefficient $\left({ }^{* *} p<0.0001\right.$, one way ANOVA followed by Tukey's test). Note that full recovery was never obtained after removing GABA (up to $2 \mathrm{~h}$ ). Error bars indicate SEM.

for instantaneous information transfer between developing neurons (Bem et al., 2002), thus, allowing common developmental regulations through activity-dependant mechanisms. More recently, it has been suggested that this widespread electrical coupling may allow the expression of structured embryonic activities before the developmental onset of chemical synaptic transmission which is concomitant with the decrease in electrical coupling (Szabo et al., 2004; Arumugam et al., 2005, Dupont et al., 2006). Here we show that the decrease of electrical coupling during de- velopment may allow for the specification of multiple small distinct networks from a single large embryonic network as suggested in previous modeling studies (Bem et al., 2002). This developmental mechanism may be useful in understanding further the ontogeny of neural networks in other species and brain regions in which the developmental decrease in electrical coupling has been well documented (Walton and Navarrete, 1991; Montoro and Yuste, 2004).

\section{References}

Albert J, Lingle CJ, Marder E, O’Neil MB (1986) A GABA-activated chloride-conductance not blocked by picrotoxin on spiny lobster neuromuscular preparations. $\mathrm{Br} \mathrm{J}$ Pharmacol 87:771-779.

Allain AE, Meyrand P, Branchereau P (2005) Ontogenic changes of the spinal GABAergic cell population are controlled by the serotonin (5-HT) system: implication of 5-HT1 receptor family. J Neurosci 25:8714-8724.

Arumugam H, Liu X, Colombo PJ, Corriveau RA, Belousov AB (2005) NMDA receptors regulate developmental gap junction uncoupling via CREB signaling. Nat Neurosci 8:1720-1726.

Bem T, Le Feuvre Y, Simmers J, Meyrand P (2002) Electrical coupling can prevent expression of adult-like properties in an embryonic neural circuit. J Neurophysiol 87:538-547.

Ben-Ari Y (2002) Excitatory actions of GABA during development: the nature of the nurture. Nat Rev Neurosci 3:728-739.

Blitz DM, Christie AE, Coleman MJ, Norris BJ, Marder E, Nusbaum MP (1999) Different proctolin neurons elicit distinct motor patterns from a multifunctional neuronal network. J Neurosci 19:5449-5463.

Casasnovas B, Meyrand P (1995) Functional differentiation of adult neural circuits from a single embryonic network. J Neurosci 15:5703-5718.

Cazalets JR, Cournil I, Geffard M, Moulins M (1987) Suppression of oscillatory activity in crustacean pyloric neurons: implication of GABAergic inputs. J Neurosci 7:2884-2893.

Charmantier G, Charmantier-Daures M, Aiken DE (1991) Metamorphosis in the lobster Homarus (Decapoda): a review. J Crust Biol 11:481-495.

Combes D, Meyrand P, Simmers J (1999) Dynamic restructuring of a rhythmic motor program by a single mechanoreceptor neuron in lobster. J Neurosci 19:3620-3628.

Cournil I, Meyrand P, Moulins M (1990) Identification of all GABA-immunoreactive neurons projecting to the lobster stomatogastric ganglion. J Neurocytol 19:478-493.

Dupont E, Hanganu IL, Kilb W, Hirsch S, Luhmann HJ (2006) Rapid developmental switch in the mechanisms driving early cortical columnar networks. Nature 439:79-83.

Faumont S (1999) Mécanismes cellulaires impliqués dans la sélection de programmes moteurs multiples par un même système de contrôle descendant: approches électrophysiologique, pharmacologique et immunohistochimique. PhD thesis, Université Bordeaux I.

Faumont S, Combes D, Meyrand P, Simmers J (2005) Reconfiguration of 
multiple motor networks by short- and long-term actions of an identified modulatory neuron. Eur J Neurosci 22:2489-2502.

Fenelon VS, Casasnovas B, Faumont S, Meyrand P (1998) Ontogenetic alteration in peptidergic expression within a stable neuronal population in lobster stomatogastric nervous system. J Comp Neurol 399:289-305.

Golan H, Grossman Y (1996) Block of glutamate decarboxylase decreases GABAergic inhibition at the crayfish synapses: possible role of presynaptic metabotropic mechanisms. J Neurophysiol 75:2089-2098.

Harris-Warrick RM, Marder E (1991) Modulation of neural networks for behavior. Annu Rev Neurosci 14:39-57.

Helluy SM, Beltz BS (1991) Embryonic development of the American lobster (Homarus americanus): quantitative staging and characterization of an embryonic molt cycle. Biol Bull 180:355-371.

Herbison AE, Heavens RP, Dyer RG (1990) Endogenous release of gammaaminobutyric acid from the medial preoptic area measured by microdialysis in the anaesthetised rat. J Neurochem 55:1617-1623.

Kaila K, Voipio J (1987) Postsynaptic fall in intracellular pH induced by GABA-activated bicarbonate conductance. Nature 330:163-165.

Kandler K (1997) Coordination of neuronal activity by gap junctions in the developing neocortex. Semin Cell Dev Biol 8:43-51.

Kandler K, Katz LC (1995) Neuronal coupling and uncoupling in the developing nervous system. Curr Opin Neurobiol 5:98-105.

Kapetanovic IM, Yonekawa WD, Torchin CD, Kupferberg HJ (1988) Effects of pharmacological manipulations on basal and newly synthesized levels of GABA, glutamate, aspartate and glutamine in mouse brain cortex. Biochem Pharmacol 37:4445-4449.

Karlsson A, Fonnum F, Malthe-Sorenssen D, Storm-Mathisen J (1974) Effect of the convulsive agent 3-mercaptopropionic acid on the levels of GABA, other amino acids and glutamate decarboxylase in different regions of the rat brain. Biochem Pharmacol 23:3053-3061.

Lamar C (1970) Mercaptopropionic acid: a convulsant that inhibits glutamate decarboxylase. J Neurochem 17:165-170.

Le Feuvre Y, Fenelon VS, Meyrand P (1999) Central inputs mask multiple adult neural networks within a single embryonic network. Nature 402:660-664

Le Feuvre Y, Fenelon VS, Meyrand P (2001) Ontogeny of modulatory inputs to motor networks: early established projection and progressive neurotransmitter acquisition. J Neurosci 21:1313-1326.

Lieske SP, Thoby-Brisson M, Telgkamp P, Ramirez JM (2000) Reconfiguration of the neural network controlling multiple breathing patterns: eupnea, sighs and gasps. Nat Neurosci 3:600-607.

Löscher W, Bohme G, Muller F, Pagliusi S (1985) Improved method for isolating synaptosomes from 11 regions of one rat brain: electron microscopic and biochemical characterization and use in the study of drug effects on nerve terminal gamma-aminobutyric acid in vivo. J Neurochem 45:879-889.

Marder E (1974) Acetylcholine as an excitatory neuromuscular transmitter in the stomatogastric system of the lobster. Nature, 251:730-731.

Marder E, Eisen JS (1984) Transmitter identification of pyloric neurons: electrically coupled neurons use different transmitters. J Neurophysiol 51:1345-1361.

Marder E, Rehm KJ (2005) Development of central pattern generating circuits. Curr Opin Neurobiol 15:86-93.

Meyrand P, Simmers J, Moulins M (1991) Construction of a patterngenerating circuit with neurons of different networks. Nature 351:60-63.

Meyrand P, Faumont S, Simmers J, Christie AE, Nusbaum MP (2000) Species-specific modulation of pattern-generating circuits. Eur J Neurosci 12:2585-2596.
Miwa A, Ui M, Kawai N (1990) G protein is coupled to presynaptic glutamate and GABA receptors in lobster neuromuscular synapse. J Neurophysiol 63:173-180.

Mizrahi A, Dickinson PS, Kloppenburg P, Fenelon V, Baro DJ, HarrisWarrick RM, Meyrand P, Simmers J (2001) Long-term maintenance of channel distribution in a central pattern generator neuron by neuromodulatory inputs revealed by decentralization in organ culture. J Neurosci 21:7331-7339.

Montoro RJ, Yuste R (2004) Gap junctions in developing neocortex: a review. Brain Res Brain Res Rev 47:216-226.

Nagy F, Cardi P, Cournil I (1994) A rhythmic modulatory gating system in the stomatogastric nervous system of Homarus gammarus. I. Pyloricrelated neurons in the commissural ganglia. J Neurophysiol 71:2477-2489.

Pearlstein E, Watson AH, Bevengut M, Cattaert D (1998) Inhibitory connections between antagonistic motor neurons of the crayfish walking legs. J Comp Neurol 399:241-254.

Peracchia C (1990) Effects of caffeine and ryanodine on low pHi-induced changes in gap junction conductance and calcium concentration in crayfish septate axons. J Membr Biol 117:79-89.

Perkins KL, Wong RK (1996) Ionic basis of the postsynaptic depolarizing GABA response in hippocampal pyramidal cells. J Neurophysiol 76:3886-3894.

Pflüger HJ (1999) Neuromodulation during motor development and behavior. Curr Opin Neurobiol 9:683-689.

Piccolino M, Neyton J, Witkovsky P, Gerschenfeld HM (1982) $\gamma$-Aminobutyric acid antagonists decrease junctional communication between L-horizontal cells of the retina. Proc Natl Acad Sci USA 79:3671-3675.

Prinz AA, Bucher D, Marder E (2004) Similar network activity from disparate circuit parameters. Nat Neurosci 7:1345-1352.

Richards KS, Marder E (2000) The actions of crustacean cardioactive peptide on adult and developing stomatogastric ganglion motor patterns. J Neurobiol 44:31-44.

Richards KS, Miller WL, Marder E (1999) Maturation of lobster stomatogastric ganglion rhythmic activity. J Neurophysiol 82:2006-2009.

Shinohara K, Hiruma H, Funabashi T, Kimura F (2000) GABAergic modulation of gap junction communication in slice cultures of the rat suprachiasmatic nucleus. Neuroscience 96:591-596.

Sillar KT, Wedderburn JF, Simmers AJ (1992) Modulation of swimming rhythmicity by 5 -hydroxytryptamine during post-embryonic development in Xenopus laevis. Proc Biol Sci 250:107-114.

Spitzer NC, Kingston PA, Manning TJ, Conklin MW (2002) Outside and in: development of neuronal excitability. Curr Opin Neurobiol 12:315-323.

Sullivan JM, Faumont S, Le Feuvre Y, Simmers J, Fenelon VS, Meyrand P (2002) Expression of embryonic-like motor patterns in an adult system: a role for histaminergic transmission. Soc Neurosci Abstr 32:367.16.

Swensen AM, Golowasch J, Christie AE, Coleman MJ, Nusbaum MP, Marder E (2000) GABA and responses to GABA in the stomatogastric ganglion of the crab Cancer borealis. J Exp Biol 203:2075-2092.

Szabo TM, Faber DS, Zoran MJ (2004) Transient electrical coupling delays the onset of chemical neurotransmission at developing synapses. J Neurosci 24:112-120.

Walton KD, Navarrete R (1991) Postnatal changes in motoneurone electrotonic coupling studied in the in vitro rat lumbar spinal cord. J Physiol (Lond) 433:283-305. 